\title{
CONVERGENCE TO EQUILIBRIUM OF BIASED PLANE PARTITIONS
}

\author{
PIETRO CAPUTO, FABIO MARTINELLI, AND FABIO LUCIO TONINELLI
}

\begin{abstract}
We study a single-flip dynamics for the monotone surface in $(2+1)$ dimensions obtained from a boxed plane partition. The surface is analyzed as a system of non-intersecting simple paths. When the flips have a non-zero bias we prove that there is a positive spectral gap uniformly in the boundary conditions and in the size of the system. Under the same assumptions, for a system of size $M$, the mixing time is shown to be of order $M$ up to logarithmic corrections.
\end{abstract}

2000 Mathematics Subject Classification: 60K35, 82C20

Keywords: Spectral gap, Mixing time, Coupling, Lozenge tiling, Plane partitions, Non-intersecting paths.

\section{Introduction, MODEL AND RESUlts}

Consider a surface in $2+1$ dimensions defined by non-negative integer heights $\ell_{x, y}$, where $x, y \in \mathbb{Z}_{+}$, such that $\ell_{x, y} \geqslant \ell_{x+1, y}$ and $\ell_{x, y} \geqslant \ell_{x, y+1}$ for all $x, y \in \mathbb{Z}_{+}$. When $\ell_{x, y}=0$ for all but finitely many $x, y$ this is called a plane partition, the two-dimensional generalization of an ordinary partition (Young diagram). When the surface is such that $\ell_{x, y} \leqslant c$ and $\ell_{x, y}=0$ when either $x \geqslant a$ or $y \geqslant b$, for some integers $a, b, c$, then it defines a boxed plane partition, or a plane partition in the box $a \times b \times c$. As we shall see, a convenient representation of a boxed plane partition is obtained by considering a system of non-intersecting simple lattice paths. Other well known equivalent characterizations are the perfect matchings (or dimers) configurations on a subgraph of the honeycomb lattice and the lozenge tilings of an hexagon.

A continuous time flip dynamics of a plane partition in the box $a \times b \times c$ is defined as follows: Every point $(x, y)$ in the rectangle $R_{a, b}=\{0, \ldots, a-1\} \times\{0, \ldots, b-1\}$ is equipped with an independent, rate 1 , Poisson clock. When $(x, y)$ rings we flip an independent $\{0,1\}$ coin $X$; if $X=1$ we replace $\ell_{x, y}$ by $\ell_{x, y}^{\prime}=\ell_{x, y}+1$ if allowed; if $X=0$ we replace $\ell_{x, y}$ by $\ell_{x, y}^{\prime}=\ell_{x, y}-1$ if allowed. When the coin is unbiased the flip dynamics converges to the uniform distribution over plane partitions in the box $a \times b \times c$. The latter is known to exhibit non trivial limiting shape or "arctic circle" phenomena in the limit of large parameters $a, b, c, \mathrm{cf}$. [3], 7] and references therein. We refer to [2] for the connection with the Wulff crystal in the low temperature 3D Ising model. It is an interesting open problem to determine the speed of convergence to the uniform equilibrium measure. We refer to [9] and references therein for the various polynomial bounds known so far and for the conjectured diffusive behavior of the spectral gap.

In this paper we shall analyze the biased case. When $X=1$ with probability $p \neq \frac{1}{2}$ then the flip dynamics converges to the probability measure $\mu_{\alpha}$ on plane partitions in the box $a \times b \times c$ such that every configuration $\ell$ has a weight proportional to $e^{-2 \alpha \operatorname{Vol}(\ell)}$, 
where $\operatorname{Vol}(\ell)$ stands for the volume $\sum_{x, y} \ell_{x, y}$ under the surface, and $e^{-2 \alpha}=p /(1-p)$. By symmetry, $\mu_{\alpha}$ is equivalent to $\mu_{-\alpha}$ and we shall restrict to positive values of $\alpha$ (or $p<1 / 2$ ). In this model there is no critical value of $\alpha$ and one has a localized surface (i.e., $\operatorname{Vol}(\ell)=O(1)$ ) for all $\alpha>0$. This follows from the fact that the number of plane partitions $\ell$ such that $\operatorname{Vol}(\ell)=v$ is $e^{O\left(v^{2 / 3}\right)}$, see Section 2 for more details.

It has been recently shown that a direct coupling argument allows to prove that, if $\alpha$ is sufficiently large, then uniformly in the size of the box one has a positive spectral gap and a mixing time of order $M=\max \{a, b, c\}$, see [6]. Below we shall prove that this actually holds for all $\alpha>0$, up to a logarithmic correction in the mixing time upper bound. As in [6] we use path-coupling arguments with an exponentially weighted metric. However, in the case of small $\alpha>0$ these arguments can only be applied to suitable coarse-grained versions of the process. In the proof of the spectral gap estimate we compare the single-flip process to two auxiliary coarse-grained dynamics. Another novel ingredient is a roughly deterministic description of the process at large scales which allows to obtain the mixing time estimate.

It is worth pointing out that the measure $\mu_{\alpha}, \alpha>0$, has a natural extension to infinite boxes, i.e. as a measure on plane partitions without any box constraint. Our spectral gap estimates implies that for all $\alpha>0$ this extended measure has a positive spectral gap.

1.1. The model. We first formulate the model in terms of configurations of nonintersecting paths and then describe the mapping needed to obtain boxed plane partitions.

Let $k, n, h$ be integers, such that $k, n \geqslant 1$ and $h \in\{-n, \ldots, n\}$. We consider the set $\Omega_{k, n}^{h}$ of $k$ polymers of length $n$, described as follows. Each polymer is a simple random walk path which starts at height 0 and ends at height $h$, and the paths are ordered: a configuration $\eta \in \Omega_{k, n}^{h}$ is characterized by integer heights $\eta_{x}^{(j)} \in\{-n, \ldots, n\}$, $j=1, \ldots, k$ and $x=0, \ldots, n$ with the constraints:

$$
\begin{gathered}
\eta_{0}^{(j)}=0, \quad \eta_{n}^{(j)}=h, \quad \nabla \eta^{(j)}(x):=\eta_{x+1}^{(j)}-\eta_{x}^{(j)} \in\{-1,+1\}, \text { and } \\
\eta_{x}^{(j)} \geqslant \eta_{x}^{(j+1)}, \quad 1 \leqslant j \leqslant k-1,0 \leqslant x \leqslant n-1 .
\end{gathered}
$$

Given $\alpha>0$, the equilibrium measure $\mu=\mu_{k, n, h}^{\alpha}$ is defined by

$$
\mu(\eta)=\frac{\exp \left(\alpha \sum_{i=1}^{k} \sum_{x=0}^{n} \eta_{x}^{(i)}\right)}{Z}, \quad \eta \in \Omega_{k, n}^{h},
$$

where

$$
Z=Z_{k, n, h}^{\alpha}=\sum_{\eta \in \Omega_{k, n}^{h}} \exp \left(\alpha \sum_{i=1}^{k} \sum_{x=0}^{n} \eta_{x}^{(i)}\right)
$$

is the normalizing constant.

For every $\xi, \sigma \in \Omega_{1, n}^{h}$, we will write simply $\xi \geqslant \sigma$ when $\xi_{x} \geqslant \sigma_{x}, 0 \leqslant x \leqslant n$. For a given pair $\xi \geqslant \sigma$ we consider the subsets

$$
E_{\xi, \sigma}=\left\{\eta \in \Omega_{k, n}^{h}, \xi \geqslant \eta^{(1)} \text { and } \eta^{(k)} \geqslant \sigma\right\} .
$$


We call $\xi$ and $\sigma$ the ceiling and the floor respectively, for intuitive reasons, see Figure 1. Given a ceiling $\xi$ and a floor $\sigma(\xi \geqslant \sigma)$ we define the equilibrium measure $\mu^{\xi, \sigma}$ by conditioning on $E_{\xi, \sigma}$ :

$$
\mu^{\xi, \sigma}=\mu\left(\cdot \mid E_{\xi, \sigma}\right) .
$$

Note that we may write more explicitly

$$
\mu^{\xi, \sigma}(\eta)=\frac{e^{-2 \alpha V^{\xi}(\eta)} \mathbf{1}_{\left\{\eta \in E_{\xi, \sigma}\right\}}}{\hat{Z}_{\xi, \sigma}}
$$

where

$$
V^{\xi}(\eta):=\sum_{1 \leqslant j \leqslant k} \sum_{k \leqslant n-1} \frac{\xi_{x}-\eta_{x}^{(j)}}{2}
$$

is the "total volume between $\xi$ and $\eta$ ", and

$$
\hat{Z}_{\xi, \sigma}:=\sum_{\eta \in E_{\xi, \sigma}} e^{-2 \alpha V^{\xi}(\eta)}
$$

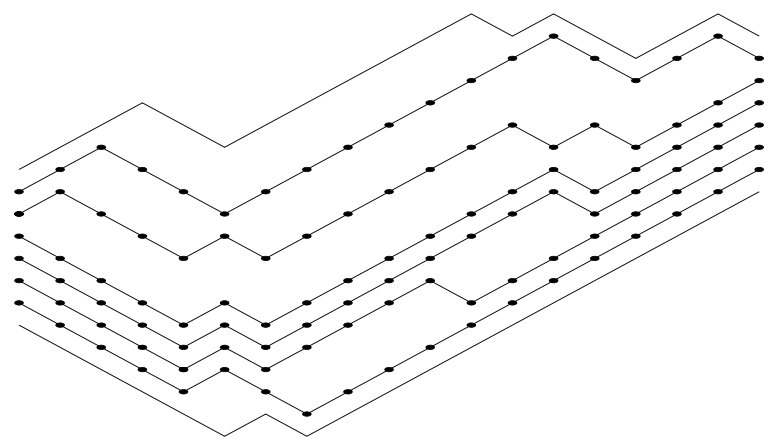

Figure 1. A configuration $\eta \in E_{\xi, \sigma}$. Here $k=6, n=18, h=6$. For graphical convenience $\eta^{(i)}$, the $i$-th component of $\eta$, has been shifted by $-i$ units in the vertical direction. The top path is the ceiling $\xi$ while the bottom path is the floor $\sigma$ shifted by $-(k+1)$ units.

We will denote by $\wedge:=\wedge^{(n, h)}$ the maximal one-polymer configuration in $\Omega_{1, n}^{h}$ :

$$
\wedge_{x}:= \begin{cases}x & \text { for } \quad 0 \leqslant x \leqslant(n+h) / 2 \\ (n+h)-x & \text { for } \quad(n+h) / 2 \leqslant x \leqslant n .\end{cases}
$$

We will also use the notation $\vee:=\vee^{(n, h)}$ for the minimal one-polymer configuration:

$$
\vee_{x}:= \begin{cases}-x & \text { for } \quad 0 \leqslant x \leqslant(n-h) / 2 \\ (h-n)+x & \text { for } \quad(n-h) / 2 \leqslant x \leqslant n .\end{cases}
$$

Note that $(n+h) / 2$ is an integer since $n$ and $h$ have the same parity, and that $\vee=-\wedge$ if $h=0$. 
1.1.1. From non-intersecting paths to plane partitions. Suppose first that $\xi=\wedge$ and $\sigma=\vee$ so that there is no further constraint on $\eta \in \Omega_{k, n}^{h}$. Then there is a bijection between $\Omega_{k, n}^{h}$ and the set of plane partitions in the box $a \times b \times c$ with $c=k, a+b=n$, $b-a=h$ (i.e. $a=(n-h) / 2, b=(n+h) / 2)$. The map is best explained informally as follows. From the configuration $\eta$ we get the stepped surface $\ell(\eta)$ by adding layers of height 1 to the basis rectangle $R_{a, b}$, according to the paths $\eta^{(j)}$ chosen in reverse order. Namely, $\eta^{(k)}$ is the first layer. On top of that we put the second layer $\eta^{(k-1)}$ and so on. This defines a bijection, see also Section 4.1.1 for more details.

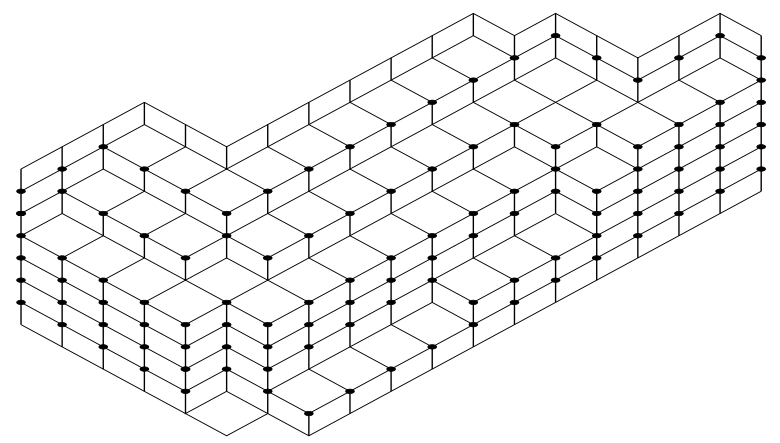

FiguRE 2. The stepped surface corresponding to the paths in Figure 1.

For any $\xi \in \Omega_{1, k}^{h}$, let $\hat{\xi} \in \Omega_{k, n}^{h}$ denote the configuration such that $\eta^{(i)}=\xi, i=1, \ldots, k$. Also, let $\ell(\hat{\xi})$ denote the associated stepped surface. Then it is easily seen that, for any $\xi \geqslant \sigma$, the map described above gives a bijection between the set $E_{\xi, \sigma}$ and the set of plane partitions $\ell$ in the box $a \times b \times c$ (as above) such that $\ell(\hat{\xi}) \leqslant \ell \leqslant \ell(\hat{\sigma})$, see Figure 2. Note that here inequalities are reversed with respect to the polymer representation.

1.2. Results. The following heat bath dynamics for configurations of lattice paths is easily checked to be equivalent to the flip dynamics discussed in the introduction. We define the continuous time Markov chain on the set $E_{\xi, \sigma}$, for a given pair $\xi, \sigma \in \Omega_{1, n}^{h}$, $\xi \geqslant \sigma$, as follows. At each $(i, x), i=1, \ldots, k$ and $x=1, \ldots, n-1$, there is an independent rate 1 Poisson clock. When $(i, x)$ rings we update the height $\eta_{x}^{(i)}$ with a new height $\widetilde{\eta}_{x}^{(i)}$ sampled according to the conditional distribution

$$
\mu_{i, x}^{\eta}(\cdot):=\mu^{\xi, \sigma}\left(\cdot \mid \eta_{x-1}^{(i)}, \eta_{x+1}^{(i)}, \eta_{x}^{(i+1)}, \eta_{x}^{(i-1)}\right),
$$

where $\eta^{(0)}=\xi$ and $\eta^{(k+1)}=\sigma$. The Dirichlet form of this process is given by

$$
\mathcal{E}(f)=\sum_{i=1}^{k} \sum_{x=1}^{n-1} \mu^{\xi, \sigma}\left[\operatorname{Var}_{i, x}(f)\right],
$$

where $\operatorname{Var}_{i, x}(f)$ denotes the function

$$
E_{\xi, \sigma} \ni \eta \rightarrow \operatorname{Var}_{i, x}^{\eta}(f):=\mu_{i, x}^{\eta}\left(f^{2}\right)-\mu_{i, x}^{\eta}(f)^{2},
$$


and $f: \Omega_{k, n}^{h} \rightarrow \mathbb{R}$ denotes an arbitrary function. The spectral gap is given by

$$
\operatorname{gap}(\alpha)=\min _{f} \frac{\mathcal{E}(f)}{\operatorname{Var}(f)},
$$

where $\operatorname{Var}(f)=\mu^{\xi, \sigma}\left(f^{2}\right)-\mu^{\xi, \sigma}(f)^{2}$, and the minimum ranges over all $f: \Omega_{k, n}^{h} \rightarrow \mathbb{R}$ such that $\operatorname{Var}(f) \neq 0$.

Theorem 1.1. For any $\alpha>0$, there exists $c(\alpha)>0$ such that, uniformly in $k, n \in \mathbb{N}$, $|h| \leqslant n$ and $\xi \geqslant \sigma \in \Omega_{1, n}^{h}$ :

$$
\operatorname{gap}(\alpha) \geqslant c(\alpha) .
$$

The estimate in Theorem 1.1 is already known to hold when $k=1$ (see [4, Th. 4.3] or, for an alternative proof, [6]).

Our second result concerns the mixing time of the Markov chain which, we recall, is defined as

$$
T_{\text {mix }}=\inf \left\{t>0: \max _{\eta \in \Omega_{k, n}^{h}}\left\|P_{t}(\eta, \cdot)-\mu\right\|_{\mathrm{var}} \leqslant 1 /(2 e)\right\},
$$

with $\|\cdot\|_{\text {var }}$ denoting the total variation norm:

$$
\left\|\nu-\nu^{\prime}\right\|_{\mathrm{var}}:=\frac{1}{2} \sum_{\eta \in \Omega_{k, n}^{h}}\left|\nu(\eta)-\nu^{\prime}(\eta)\right| .
$$

$P_{t}(\eta, \cdot)$ is the law, at time $t$, of the Markov chain started from $\eta$ at time zero.

Theorem 1.1 implies that the mixing time of the Markov chain defined above in the case $2 k=n, h=0, \xi=\wedge, \sigma=\vee$ is $O\left(n^{3}\right)$. This is a simple consequence of the well-known inequality

$$
T_{\text {mix }} \leqslant \operatorname{gap}^{-1}\left(1-\log \left(\min _{\eta} \mu(\eta)\right)\right),
$$

(see also Lemma 4.3 below). We can however prove:

Theorem 1.2. Let $M=\max (n, k)$ and $c=6$. For every $\alpha>0$ there exists $C(\alpha)<\infty$ such that uniformly in the choice of ceiling $\xi$ and floor $\sigma, T_{\operatorname{mix}} \leqslant C(\alpha) M(\log M)^{c}$.

We mention that the conjectured behavior is $O(M)$, without logarithmic corrections. This bound is known to hold if $\alpha$ is sufficiently large [6.

For simplicity we have stated these results for a positive constant bias $\alpha>0$, but there is no difficulty to extend them to the case of a non-homogeneous bias $\alpha_{x, y}$ on each column of the stepped surface $\ell_{x, y}$, provided that there exists $\alpha_{0}>0$ such that $\alpha_{x, y} \geqslant \alpha_{0}$ for all $x, y$. Alternatively, one could place a non-homogeneous bias $\alpha_{(i, x)}$ associated to each polymer $i$ and position $x$ in the definition of the measure (1.2).

The rest of the paper is organized as follows. In Section 2 we provide some preliminaries and prove a couple of key equilibrium estimates to be used in the proof of the main theorems. The latter is given in Section 3 (Theorem 1.1) and Section 4 (Theorem 1.2). 


\section{Preliminaries}

2.1. Particles and vacancies. Each polymer $\eta^{(j)}$ can be characterized by the positions of its positive increments, also called particles. More precisely, for every $1 \leqslant j \leqslant k$, let $x_{i}^{(j)}=x_{i}^{(j)}(\eta), i \geqslant 1$ denote the position of the $i$-th positive increment in the $j$-th polymer, defined recursively by:

$$
\begin{gathered}
x_{1}^{(j)}=\min \left\{x \in\{0, \ldots, n-1\}: \nabla \eta^{(j)}(x)=+1\right\}, \ldots \\
x_{\ell+1}^{(j)}=\min \left\{x>x_{\ell}^{(j)}: \nabla \eta^{(j)}(x)=+1\right\} .
\end{gathered}
$$

Note that, given $h$, the $k$ polymers in the configuration $\eta \in \Omega_{k, n}^{h}$ all have the same number $N=N(h, n)=(n+h) / 2$ of particles (they all have the same length $n$, the same starting point 0 and the same end point $h$ ). Observe that, because of the order constraint, particles obey the following relations: $x_{i}^{(j)} \leqslant x_{i}^{(j+1)}, i=1, \ldots, N$ and $j=1, \ldots, k-1$. We often write $\mathbf{x}$ or $\mathbf{x}(\eta)$ for the collection of particle positions of a given configuration $\eta$.

The set of vacancies for the polymer $\eta^{(j)}$ is defined as the set of points in $\{0, \ldots, n-1\}$ which do not contain particles. Of course, the number of particles plus the number of vacancies for $\eta \in \Omega_{1, n}^{h}$ equals $n$.

2.2. Monotonicity. Trajectories of the Markov chain corresponding to distinct initial conditions and/or distinct boundary constraints can be realized on the same probability space by a standard coupling argument. This is a straightforward generalization of the argument for a single polymer, see [5, Section 2]. It follows that the Markov chain enjoys the following useful monotonicity property. If $\eta^{\xi, \sigma}(t ; \zeta)$ denotes the evolution of the surface with ceiling $\xi$ and floor $\sigma$ at time $t$ and with starting configuration $\zeta$ at time 0 , then almost surely one has

$$
\eta^{\xi, \sigma}(t ; \zeta) \geqslant \eta^{\xi^{\prime}, \sigma^{\prime}}\left(t ; \zeta^{\prime}\right),
$$

whenever $\xi \geqslant \xi^{\prime}, \sigma \geqslant \sigma^{\prime}$ and $\zeta \geqslant \zeta^{\prime}$. Here for two systems of polymers $\eta, \zeta \in \Omega_{k, n}^{h}$ we use the convention that $\eta \geqslant \zeta$ means $\eta^{(i)} \geqslant \zeta^{(i)}$ for all $i$.

Let $\mathbb{E}$ denote expectation with respect to this global coupling $\mathbb{P}$. Using the notation $\mathbb{E}\left[f\left(\eta^{\xi, \sigma}(t ; \zeta)\right)\right]=P_{t} f(\zeta), f: \Omega_{k, n}^{h} \rightarrow \mathbb{R}$, then (2.1) implies that for every fixed $t \geqslant 0$, the function $P_{t} f$ is increasing whenever $f$ is increasing, where a function $f$ is called increasing if $f(\eta) \geqslant f(\zeta)$ for any $\eta, \zeta$ such that $\eta \geqslant \zeta$. Useful inequalities for the equilibrium measures can be derived from this. For instance, taking the limit $t \rightarrow \infty$ in (2.1) yields the inequality

$$
\mu^{\xi, \sigma}(f) \geqslant \mu^{\xi^{\prime}, \sigma^{\prime}}(f)
$$

for any increasing $f$ and any $\xi \geqslant \xi^{\prime}, \sigma \geqslant \sigma^{\prime}$. We will often use one form or another of the inequality (2.2) without explicit reference.

2.3. Tightness of the excess volume and decay of correlation. Here we prove some equilibrium results concerning the exponential decay of spatial correlations, and the exponential tightness of the "excess volume" $V^{\xi}(\eta)$. While our main aim is to provide the necessary tools for the proof of Theorems 1.1 and 1.2, such results may be of independent interest. 
We start with a basic estimate for the case where $h=0, n \in 2 \mathbb{N}$ and the ceiling is the maximal possible configuration, $\wedge$.

Lemma 2.1. Let $h=0$. For every $\alpha>0$ there exists $c_{1}(\alpha)>0$ such that, uniformly in $n \in 2 \mathbb{N}, k \in \mathbb{N}$ and in the configuration $\sigma \in \Omega_{1, n}^{0}$ of the floor,

$$
\mu^{\wedge, \sigma}\left(V^{\wedge}(\eta) \geqslant i\right) \leqslant e^{-c_{1}(\alpha) i}
$$

for every $i \in \mathbb{N}$. In particular, there exists $p(\alpha)>0$ such that

$$
\mu^{\wedge, \sigma}\left(\eta^{(j)}=\wedge, \forall 1 \leqslant j \leqslant k\right) \geqslant p(\alpha) .
$$

Proof. By monotonicity, we can upper bound the probability in (2.3) replacing $\sigma$ with $\vee$ :

$$
\mu^{\wedge, \sigma}\left(V^{\wedge}(\eta) \geqslant i\right) \leqslant \mu^{\wedge, \vee}\left(V^{\wedge}(\eta) \geqslant i\right) .
$$

Always by monotonicity, the right-hand side of (2.5) is non-decreasing in $n$ and $k$. Therefore,

$$
\mu^{\wedge, \sigma}\left(V^{\wedge}(\eta) \geqslant i\right) \leqslant \frac{\sum_{v \geqslant i} e^{-2 \alpha v} \mathcal{N}(v)}{\sum_{v \geqslant 0} e^{-2 \alpha v} \mathcal{N}(v)}
$$

where $\mathcal{N}(v)$ is the number of plane partitions of volume $v$, and the right-hand side is just the limit $n \rightarrow \infty, k \rightarrow \infty$ of the right-hand side of (2.5). The dependence on $\sigma, k$ and $n$ has then disappeared. Since

$$
\mathcal{N}(v) \stackrel{v \rightarrow \infty}{\sim} \frac{a_{1}}{v^{25 / 36}} \exp \left(a_{2} v^{2 / 3}\right)
$$

for some (explicit) positive constants $a_{1}, a_{2}$ [10], one obtains immediately (2.3). Eq. (2.4) is obtained from (2.3) just taking $i=1$ and $p(\alpha)=1-\exp \left(-c_{1}(\alpha)\right)$.

Even if the ceiling $\xi$ does not coincide with $\wedge$, it is intuitive that the polymer $\eta^{(1)}$ gets more and more squeezed to $\xi$ when $k$ becomes large. This is one of the implications of the next result:

Lemma 2.2. For every $\alpha>0$ there exists $c_{2}(\alpha)>0$ such that the following holds. Uniformly in $\xi \geqslant \sigma \in \Omega_{1, n}^{h}$, in $n, k \in \mathbb{N},|h| \leqslant n$ and $0 \leqslant x \leqslant n$,

$$
\mu^{\xi, \sigma}\left(\eta_{x}^{(j)} \neq \xi_{x}\right) \leqslant e^{-c_{2}(\alpha)(k-j+1)} .
$$

Moreover, for every $0<a<b<n$,

$$
\mu^{\xi, \sigma}\left(\eta_{x}^{(k)} \neq \xi_{x} \forall a \leqslant x \leqslant b\right) \leqslant e^{-c_{2}(\alpha)(b-a)} .
$$

Proof. For a fixed $1 \leqslant x<n$, let $\tilde{\wedge} \in \Omega_{1, n}^{h}$ be defined as follows (see Fig. 3):

$$
\tilde{\wedge}_{y}:=\max \left(\xi_{x}-|y-x|, \vee_{y}\right), 0 \leqslant y \leqslant n .
$$

Note that $\xi \geqslant \tilde{\wedge}$ and of course $\sigma \geqslant \vee$. Then, by monotonicity and the fact that $\xi_{x}=\tilde{\Lambda}_{x}$ we have

$$
\mu^{\xi, \sigma}\left(\eta_{x}^{(j)}<\xi_{x}\right) \leqslant \mu^{\tilde{\wedge}, \vee}\left(\eta_{x}^{(j)}<\tilde{\wedge}_{x}\right) .
$$




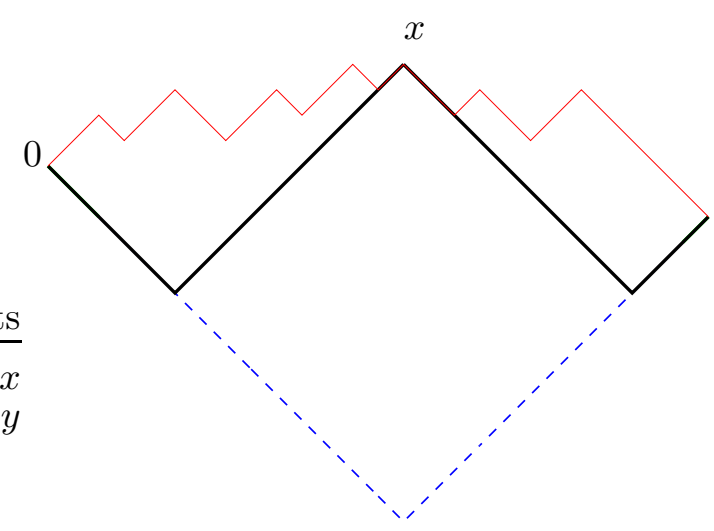

Figure 3. Graphical construction of $\tilde{\wedge}$. The thin full line denotes $\xi$, the dashed line $\vee$ and the thick full line is $\tilde{\wedge}$, for a given value of $x$. The floor $\sigma$ is not drawn, since it has no influence on the construction of $\tilde{\wedge}$.

Now, $\eta_{x}^{(j)}<\tilde{\wedge}_{x}$ implies $V^{\tilde{\wedge}}(\eta) \geqslant k-j+1$. As in Eq. (2.6) , from monotonicity it follows that

$$
\mu^{\xi, \sigma}\left(\eta_{x}^{(j)}<\xi_{x}\right) \leqslant \frac{\sum_{v \geqslant(k-j+1)} e^{-2 \alpha v} \mathcal{N}(v)}{\sum_{v \geqslant 0} e^{-2 \alpha v} \mathcal{N}(v)}
$$

and (2.8) follows from (2.7).

Next, we prove (2.9). Denote by $x_{r}^{(0)}, 1 \leqslant r \leqslant N(n, h)$ the positions of the particles of the ceiling $\xi$, and let $\mathcal{I}_{a, b}:=\left\{x_{i_{a}}^{(0)}, x_{i_{a}+1}^{(0)}, \ldots, x_{i_{b}}^{(0)}\right\}$ the set of those particles which are contained in the interval $\{a, \ldots, b-1\}$ (the cardinality of $\mathcal{I}_{a, b}$ does not exceed $(b-a)$ and can be zero). We use also the notation $x_{r}^{(k+1)}, 1 \leqslant r \leqslant N(n, h)$ to denote the positions of the particles of the floor $\sigma$. The event in the left-hand side of (2.9) implies that $x_{r}^{(k)}>x_{r}^{(0)}$ for every $i_{a} \leqslant r \leqslant i_{b}$. On the other hand, from (2.8) we know that the event $\left\{x_{i_{b}}^{(j)}=x_{i_{b}}^{(0)} \forall 1 \leqslant j \leqslant k\right\}=\left\{x_{i_{b}}^{(k)}=x_{i_{b}}^{(0)}\right\}$ has probability at least $1-\exp \left(-c_{2}(\alpha)\right)$, uniformly in all the parameters. Assume that this event is not realized. In this case, the probability that $\left\{x_{i_{b}-1}^{(j)}=x_{i_{b}-1}^{(0)} \forall 1 \leqslant j \leqslant k\right\}=\left\{x_{i_{b}-1}^{(k)}=x_{i_{b}-1}^{(0)}\right\}$ is again lower bounded by $1-\exp \left(-c_{2}(\alpha)\right)$ : indeed, by monotonicity it is sufficient to consider the case where $x_{i_{b}}^{(j)}=x_{i_{b}}^{(k+1)}$ for every $1 \leqslant j \leqslant k$, and to apply once more (2.8). Iterating this procedure, we see that the left-hand side of (2.9) is lower-bounded by

$$
e^{-c_{2}(\alpha)\left|\mathcal{I}_{a, b}\right|}
$$

One can then repeat the argument with the vacancies replacing the particles. The argument is the same except that vacancies have to be matched from left to right (while particles have been matched from right to left). Since the number of vacancies plus the number of particles in $\{a, \ldots, b\}$ equals $(b-a)$, one obtains immediately (2.9) (modulo redefining $c_{2}(\alpha)$ ). 


\section{Proof of Theorem 1.1}

To prove the spectral gap estimate we shall use a three-fold decomposition that can be roughly described as follows. The first step, carried out in Section 3.2, allows to reduce the original process to a process with a bounded (i.e. independent of $n, k$ ) number $s$ of polymers. This is achieved by estimating the spectral gap of a new process whose elementary moves consist in the updatings of sets of $s$ full-length polymers. The second step, see Section 3.1 below, allows to further reduce the problem to a process where each of the $s$ polymers has a bounded (i.e. independent of $n, k$ ) number $r$ of particles. This is obtained by analyzing a coarse-grained dynamics where we update blocks of particles. The last step is a rough estimate for a system with $s$ polymers and $r$ particles, see Section 3.3. Finally, in Section 3.4 we prove the theorem by gathering all the pieces together.

3.1. Particle block-dynamics. Let $\nu_{j, m}^{\mathbf{x}}$, for $j<m$, denote the equilibrium measure $\mu^{\xi, \sigma}$ on $E_{\xi, \sigma}$ conditioned on the values of the particle positions

$$
\left\{x_{v}^{(u)}, u=1, \ldots, k\right\}, \quad v \leqslant j, \text { and } v \geqslant m .
$$

As a convention, if $v<1$ then we set (deterministically) $x_{v}^{(j)}=0$ for all $j$. Similarly, if $v>N$, we set $x_{v}^{(j)}=N$. Recall that $N=N(h, n)$ denotes the total number of particles.

It will be convenient to have the following alternative notation. For a fixed integer $\ell$ we define the measures $\rho_{i, \ell}^{\mathbf{x}}:=\nu_{i-\ell-1, i+\ell+1}^{\mathbf{x}}$. Once the values of (3.1) are given, $\rho_{i, \ell}^{\mathbf{x}}$ is a distribution on the portion of paths $\eta^{(j)}$ in the segments $\left\{a^{(j)}+1, \ldots, b^{(j)}-1\right\}$, $j=1, \ldots, k$, where $a^{(j)}:=x_{i-\ell-1}^{(j)}+1, b^{(j)}:=x_{i+\ell+1}^{(j)}$, see Figure 4.

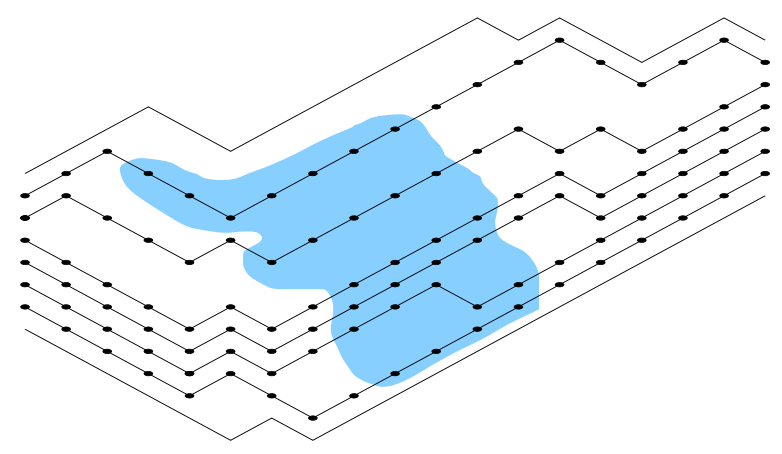

Figure 4. An illustration of the definition of the measure $\rho_{i, \ell}^{\mathbf{x}}$ for the paths from Figure 1. Here $\ell=2, i=5$. The shaded region is the portion of paths distributed according to $\rho_{i, \ell}^{\mathrm{x}}$, while the rest of the configuration is frozen.

We will study the following Markov chain with state space $E_{\xi, \sigma}$, for a given pair $\xi, \sigma \in \Omega_{1, n}^{h}$, with $\xi \geqslant \sigma$. This auxiliary process, which we call the particle blockdynamics, is reversible w.r.t. the equilibrium measure $\mu^{\xi, \sigma}$.

We have $N(h, n)$ independent Poisson clocks with parameter 1 . When the $i$-th clock rings we consider the current configuration $\eta$, and update the portion of paths $\eta^{(j)}$ in 
the segments $\left\{a^{(j)}+1, \ldots, b^{(j)}-1\right\}, j=1, \ldots, k$ with a sample from the conditional distribution $\rho_{i, \ell}^{\mathbf{x}}$. The rest of the configuration $\eta$ is left unchanged. In other words, we are removing from the system all particles at positions $x_{i-\ell}^{(j)}, \ldots, x_{i+\ell}^{(j)}, j=1, \ldots, k$ and we are replacing them by a sample from $\rho_{i, \ell}^{\mathbf{x}}$.

The generator of this process can be written as

$$
\mathcal{G} f=\sum_{i=1}^{N}\left[\rho_{i, \ell}^{\mathbf{x}}(f)-f\right],
$$

where $f$ denotes a function $f: E_{\xi, \sigma} \rightarrow \mathbb{R}$ and $\rho_{i, \ell}^{\mathbf{x}}(f)$ is the function $\eta \rightarrow \int f(\zeta) \rho_{i, \ell}^{\mathbf{x}}(d \zeta)$ for $\mathbf{x}=\mathbf{x}(\eta)$. Since $\rho_{i, \ell}^{\mathbf{x}}$ are conditional expectations we see that $\mu^{\xi, \sigma}\left(f\left(\rho_{i, \ell}^{\mathbf{x}}(f)-f\right)\right)=$ $\mu^{\xi, \sigma}\left(\left(\rho_{i, \ell}^{\mathbf{x}}(f)\right)^{2}-\rho_{i, \ell}^{\mathbf{x}}\left(f^{2}\right)\right)$, so that the Dirichlet form of the process is

$$
-\mu^{\xi, \sigma}(f \mathcal{G} f)=\sum_{i=1}^{N} \mu^{\xi, \sigma}\left(\operatorname{Var}_{\rho_{i, \ell}^{\mathbf{x}}}(f)\right) .
$$

In particular, $\mathcal{G}$ is self-adjoint in $L^{2}\left(\mu^{\xi, \sigma}\right)$. Let $\operatorname{gap}(\mathcal{G})$ denote the spectral gap of this process, which of course depends on the choice of $\ell \in \mathbb{N}$.

Proposition 3.1. For any $\alpha>0$ and any $k \in \mathbb{N}$, there exists $\ell=\ell(\alpha, k)$ such that uniformly in $h, n$, and $\xi, \sigma \in \Omega_{1, n}^{h}$ we have

$$
\operatorname{gap}(\mathcal{G}) \geqslant 1 .
$$

To prove Proposition 3.1 we use a coupling argument. Consider two evolutions $\eta(t), \eta^{\prime}(t), t \geqslant 0$, of the Markov chain described above, with initial conditions $\eta$ and $\eta^{\prime}$ respectively, where $\eta, \eta^{\prime} \in E_{\xi, \sigma}$. Proposition 3.1 is an immediate consequence of Lemma 3.2 below. Indeed, a well-known argument (see e.g. Proposition 3 in [9]) shows that, for any coupling $\mathbb{P}$ of the two evolutions

$$
\operatorname{gap}(\mathcal{G}) \geqslant-\liminf _{t \rightarrow \infty} \frac{1}{t} \log \left[\max _{\eta, \eta^{\prime}} \mathbb{P}\left(\eta(t) \neq \eta^{\prime}(t)\right)\right] .
$$

Lemma 3.2. For every $\alpha>0$ and $k \in \mathbb{N}$ there exist $\ell \in \mathbb{N}, \gamma>0$ and a coupling $\mathbb{P}$ of $\left(\eta(t), \eta^{\prime}(t)\right)$ such that, uniformly in the starting configurations and uniformly in the parameters $h, n$ and $\xi, \sigma \in \Omega_{1, n}^{h}$

$$
\mathbb{P}\left(\eta(t) \neq \eta^{\prime}(t)\right) \leqslant k N(h, n) e^{\gamma n} e^{-t}, \quad t \geqslant 0 .
$$

Proof. Consider the distance

$$
d_{\gamma}\left(\eta, \eta^{\prime}\right)=\sum_{i=1}^{N} \sum_{j=1}^{k} e^{-\gamma i} 1_{\left\{x_{i}^{(j)} \neq y_{i}^{(j)}\right\}}, \quad \eta, \eta^{\prime} \in E_{\xi, \sigma},
$$

where $x_{i}^{(j)}, y_{i}^{(j)}$ denote the positions of the $i$-th particle of the $j$-th polymer in the configurations $\eta$ and $\eta^{\prime}$ respectively, and $\gamma>0$ is to be determined later. Note that the minimal non-zero value of $d_{\gamma}(\cdot, \cdot)$ is $e^{-\gamma n}$. Therefore, by Markov's inequality

$$
\mathbb{P}\left(\eta(t) \neq \eta^{\prime}(t)\right) \leqslant e^{\gamma n} \mathbb{E}\left(d_{\gamma}\left(\eta(t), \eta^{\prime}(t)\right)\right) .
$$


It remains to show that $\mathbb{E}\left(d_{\gamma}\left(\eta(t), \eta^{\prime}(t)\right)\right) \leqslant k N(h, n) e^{-t}$. In Lemma 3.4 below we show that it is sufficient to prove that

$$
\left[\widetilde{\mathcal{G}} d_{\gamma}\right]\left(\eta, \eta^{\prime}\right):=\left.\frac{d}{d t} \mathbb{E}\left(d_{\gamma}\left(\eta(t), \eta^{\prime}(t)\right)\right)\right|_{t=0^{+}} \leqslant-d_{\gamma}\left(\eta, \eta^{\prime}\right),
$$

for all pairs $\eta, \eta^{\prime}$ such that $d_{0}\left(\eta, \eta^{\prime}\right)=1$, i.e. when there is only one discrepancy in the particle configurations. In this case, $d_{\gamma}\left(\eta, \eta^{\prime}\right)=e^{-\gamma i}$ where $i$ is the label of the discrepancy.

Up to now we have not specified the coupling. If we use the same Poisson clocks for the two evolutions, the generator $\widetilde{\mathcal{G}}$ of the coupled dynamics can be written as

$$
\widetilde{\mathcal{G}}=\sum_{i=1}^{N}\left(E_{i, \ell}-1\right),
$$

where $E_{i, \ell}$ denotes a (not yet specified) coupling of the local equilibria $\rho_{i, \ell}^{\mathbf{x}}$ of the block of $2 \ell+1$ particles around the $i$-th particle for the $k$ polymers, cf. (3.2).

To prove (3.6), we may choose the coupling in such a way that if $\eta, \eta^{\prime}$ have a single discrepancy at a given particle label $i$ and at a given polymer label $j$, then $\left[E_{v, \ell} d_{\gamma}\right]\left(\eta, \eta^{\prime}\right)=0$ for all $v$ such that $v-\ell \leqslant i \leqslant v+\ell$. By construction, there are at least $\ell$ such blocks for any fixed $i$ (this is the case if e.g. $i=1$ or $i=N$ ).

Let $E_{i}^{-}=E_{i-\ell-1, \ell}$ (respectively, $E_{i}^{+}=E_{i+\ell+1, \ell}$ ) denote the coupling corresponding to the block of $2 \ell+1$ particles just to the left (resp. just to the right) of particle $i$. Note that if e.g. $i \leqslant \ell+1$ then there is no block just to the left of $i$ and we may set $E_{i}^{-}=1$ for such $i$. Similarly, if $i \geqslant N-\ell$ then there is no block just to the right of $i$ and we can set $E_{i}^{+}=1$ in this case. Since all other blocks give a trivial contribution to (3.6) we see that

$$
\begin{aligned}
& {\left[\widetilde{\mathcal{G}} d_{\gamma}\right]\left(\eta, \eta^{\prime}\right)} \\
& \quad \leqslant-\ell d_{\gamma}\left(\eta, \eta^{\prime}\right)+\left[\left(E_{i}^{-}-1\right) d_{\gamma}\right]\left(\eta, \eta^{\prime}\right)+\left[\left(E_{i}^{+}-1\right) d_{\gamma}\right]\left(\eta, \eta^{\prime}\right) .
\end{aligned}
$$

Recall that $d_{\gamma}\left(\eta, \eta^{\prime}\right)=e^{-\gamma i}$. Then we can estimate

$$
\begin{aligned}
{\left[E_{i}^{+} d_{\gamma}\right]\left(\eta, \eta^{\prime}\right) } & =\sum_{u=1}^{k} \sum_{v=i+1}^{i+2 \ell+1} e^{-\gamma v} E_{i}^{+}\left[1_{\left\{x_{v}^{(u)} \neq y_{v}^{(u)}\right\}}\right] \\
& \leqslant k e^{-\gamma i} \sum_{v=1}^{\infty} e^{-\gamma v}=\frac{k e^{-\gamma}}{1-e^{-\gamma}} d_{\gamma}\left(\eta, \eta^{\prime}\right),
\end{aligned}
$$

where we have bounded by 1 the probability of a discrepancy.

On the other hand, denoting by $F_{v}$ the event that there exists $u \in\{1, \ldots, k\}$ such that $x_{i-v}^{(u)} \neq y_{i-v}^{(u)}$, we have

$$
\begin{aligned}
{\left[E_{i}^{-} d_{\gamma}\right]\left(\eta, \eta^{\prime}\right) } & =\sum_{u=1}^{k} \sum_{v=i-2 \ell-1}^{i-1} e^{-\gamma v} E_{i}^{-}\left[1_{\left\{x_{v}^{(u)} \neq y_{v}^{(u)}\right\}}\right] \\
& \leqslant k e^{-\gamma i} \sum_{v=1}^{2 \ell+1} e^{\gamma v} E_{i}^{-}\left[1_{F_{v}}\right]=k d_{\gamma}\left(\eta, \eta^{\prime}\right) \sum_{v=1}^{2 \ell+1} e^{\gamma v} E_{i}^{-}\left[1_{F_{v}}\right]
\end{aligned}
$$


with the convention that $1_{F_{v}}=0$ if $i-v<1$.

In Lemma 3.3 below we prove that for a suitable choice of the coupling there exists $c=c(\alpha, k)>0$ independent of $\gamma$ such that that

$$
E_{i}^{-}\left[1_{F_{v}}\right] \leqslant e^{-c v}, \quad 1 \leqslant v \leqslant 2 \ell+1 .
$$

If we accept this estimate, from (3.8), (3.9) we conclude that if e.g. $\gamma=c / 2$ then (3.6) follows for $\ell$ sufficiently large (depending on $k$ and $\alpha$ ).

We turn to the proof of (3.10). Let $\mathbf{x}, \mathbf{y}$ denote the collections $\left\{x_{v}^{(u)}\right\},\left\{y_{v}^{(u)}\right\}$ of all positions of particles of two configurations $\eta, \eta^{\prime} \in E_{\xi, \sigma}$ and consider the associated probability measures $\nu_{j, m}^{\mathbf{x}}, \nu_{j, m}^{\mathbf{y}}$ defined in (3.1). For a fixed pair of integers $j, m$, let $\widetilde{\nu}=\widetilde{\nu}^{j, m}$ be the independent coupling of $\nu_{j-m-1, j}^{\mathbf{x}}$ and $\nu_{j-m-1, j}^{\mathbf{y}}$. That is, we are freezing all particles labeled $i \leqslant j-m-1$ or $i \geqslant j$ and we sample the $m$ particles labeled $i=j-m, \ldots, j-1$ according to the independent coupling of $\nu_{j-m-1, j}^{\mathbf{x}}$ and $\nu_{j-m-1, j}^{\mathbf{y}}$. We say that $\mathbf{x}, \mathbf{y}$ agree up to $j-m-1$ if $x_{v}^{(u)}=y_{v}^{(u)}$, for all $u=1, \ldots, k$ and for all $v \leqslant j-m-1$.

Lemma 3.3. There exists $\varepsilon>0$ depending only on $\alpha$ and $k$ such that, if $\mathbf{x}, \mathbf{y}$ agree up to $j-m-1$, then

$$
\widetilde{\nu}\left(x_{j-1}^{(u)}=y_{j-1}^{(u)}, \text { for all } u=1, \ldots, k\right) \geqslant \varepsilon .
$$

In particular, (3.10) holds.

Proof. Let us first show that (3.11) implies (3.10). First of all, let us sample $x_{i-1}^{(u)}, y_{i-1}^{(u)}$, $u=1, \ldots, k$, using $\widetilde{\nu}$ with $j=i$ and (supposing for simplicity $i>2 \ell+1) m=2 \ell+1$ (recall that $i$ is the index appearing in the proof of Lemma 3.2). Then (3.11) implies that there is a full matching $x_{i-1}^{(u)}=y_{i-1}^{(u)}$ for all $u=1, \ldots, k$ with probability at least $\varepsilon$. Thus (3.10) holds when $v=1$ and $e^{-c}=1-\varepsilon$. The case $v>1$ is obtained by recursion. Namely, the coupling $E_{i}^{-}$can be further defined as follows. If we have a full matching $x_{i-1}^{(u)}=y_{i-1}^{(u)}$ for all $u=1, \ldots, k$ then we can match all the remaining particles. If we do not have the full matching we sample particles labeled $i-2$ by $\widetilde{\nu}$ with $j=i-1$ and $m=2 \ell$ and a suitable choice of the values $x_{i-1}^{(u)}, y_{i-1}^{(u)}$ (the ones that were sampled in the first step). Again, if we have a full matching for particles labeled $i-2$ we can match all the remaining particles labeled $i-3, \ldots, i-m$. We repeat this procedure at later steps. Since (3.11) shows that there is a probability at least $1-e^{-c}$ to have a full matching at every step the bound (3.10) follows.

To prove (3.11) we are going to use the same argument as in the proof of Lemma 2.2. see the proof of (2.13) in particular. We first sample the pairs $x_{r}^{(1)}, y_{r}^{(1)}, r=$ $j-m, \ldots, j-1$ with an independent coupling for the corresponding marginals. Note that even if $x_{j}^{(1)} \neq y_{j}^{(1)}$ the positions $x_{j-1}^{(1)}, y_{j-1}^{(1)}$ have the same ground state (i.e. minimal position) dictated by the ceiling $\xi$ and the (common) boundary conditions for the particles labeled $1, \ldots, j-m-1$ of polymer 1 . From the proof of Lemma 2.2 we know that this implies that the matching event $x_{j-1}^{(1)}=y_{j-1}^{(1)}$ has probability at least $\delta^{2}$ where $\delta=\delta(\alpha)>0$ is the probability that $x_{j-1}^{(1)}$ equals its ground state position. Next, we 
sample $x_{r}^{(2)}, y_{r}^{(2)}, r=j-m, \ldots, j-1$ with an independent coupling of the marginals conditioned on the configuration of $\left\{x_{r}^{(1)}, y_{r}^{(1)}\right\}_{r=j-m, \ldots, j-1}$ which we extracted in the previous step. We claim that, conditionally on the occurrence of the matching $x_{j-1}^{(1)}=$ $y_{j-1}^{(1)}$, the matching event $x_{j-1}^{(2)}=y_{j-1}^{(2)}$ has probability at least $\delta^{2}$. Indeed, even if the ceilings "felt" by the two copies of the positions $x_{r}^{(2)}, y_{r}^{(2)}$ of particles of polymer 2 are in general distinct, the ground state positions for $x_{j-1}^{(2)}, y_{j-1}^{(2)}$ are dictated only by the positions $x_{j-1}^{(1)}, y_{j-1}^{(1)}$ which are now assumed to coincide. In particular the argument from the proof of Lemma 2.2 again applies. This procedure can be repeated until the last polymer is reached and in conclusion the independent coupling gives probability at least $\varepsilon=\delta^{2 k}$ to the full matching event in (3.11).

In the proof of Lemma 3.2 (cf. (3.6) ) we have used a continuous time version of the so-called path coupling argument, see [1] or e.g. [8, Theorem 14.5] for the usual discrete-time version. For the sake of completeness we give a proof in the next lemma.

Lemma 3.4. Suppose that (3.6) holds for all pairs $\eta, \eta^{\prime}$ such that $d_{0}\left(\eta, \eta^{\prime}\right)=1$. Then $\mathbb{E}\left(d_{\gamma}\left(\eta(t), \eta^{\prime}(t)\right)\right) \leqslant k N(h, n) e^{-t}$, for all $t \geqslant 0$ and for all initial data $\eta, \eta^{\prime}$.

Proof. We may define a graph having as vertices the elements of $E_{\xi, \sigma}$ by declaring a pair $\eta, \eta^{\prime}$ to be an edge whenever $d_{0}\left(\eta, \eta^{\prime}\right)=1$. For any $\eta, \eta^{\prime}$, let $\Omega\left(\eta, \eta^{\prime}\right)$ denote the set of paths connecting $\eta$ and $\eta^{\prime}$, i.e. $\omega \in \Omega\left(\eta, \eta^{\prime}\right)$ if $\omega=\left(\omega_{1}, \ldots, \omega_{r}\right), \omega_{1}=\eta, \omega_{r}=\eta^{\prime}$ and $d_{0}\left(\omega_{i}, \omega_{i+1}\right)=1, i=1, \ldots, r-1$. Then one checks that

$$
d_{\gamma}\left(\eta, \eta^{\prime}\right)=\min _{\omega \in \Omega\left(\eta, \eta^{\prime}\right)} \sum_{i=1}^{r-1} d_{\gamma}\left(\omega_{i}, \omega_{i+1}\right) .
$$

To prove (3.12) observe that it suffices to exhibit one path which achieves the equality since by construction it is clear that $d_{\gamma}\left(\eta, \eta^{\prime}\right) \leqslant \sum_{i=1}^{r-1} d_{\gamma}\left(\omega_{i}, \omega_{i+1}\right)$ for any $\omega \in \Omega\left(\eta, \eta^{\prime}\right)$. Such a path can be informally defined as follows. Consider the bottom paths $\eta^{(k)}, \eta^{(k)}$ and the positions $x$ where $\eta_{x}^{(k)}>\eta_{x}^{\prime(k)}$. Then move one by one the particles of $\eta^{(k)}$ in this region until we have $\eta_{x}^{(k)} \leqslant \eta_{x}^{\prime(k)}$ everywhere. Then consider the paths $\eta^{(k-1)}, \eta^{(k-1)}$ and the positions $x$ where $\eta_{x}^{(k-1)}>\eta_{x}^{\prime(k-1)}$. As before, move one by one the particles of $\eta^{(k-1)}$ in this region until we have $\eta_{x}^{(k-1)} \leqslant \eta_{x}^{\prime(k-1)}$ everywhere. We repeat this procedure until we reach the top paths. At this point we have reached a configuration $\widetilde{\eta}$ such that $\widetilde{\eta} \leqslant \eta$ (everywhere). Next we start from the top paths $\eta^{(1)}, \eta^{\prime(1)}$ and consider the region where $\widetilde{\eta}_{x}^{(1)}<\eta_{x}^{\prime(1)}$. We can move one by one the particles in this region until we have $\widetilde{\eta}^{(1)}=\eta^{\prime(1)}$ (everywhere). We repeat with the paths $\widetilde{\eta}^{(2)}, \eta^{\prime(2)}$ and so on until we reach the bottom polymers labeled $k$. This construction produces a path which realizes the minimum (3.12) since we never used more than the strictly necessary moves.

From the triangle inequality, for each pair of initial conditions $\eta, \eta^{\prime}$ we have

$$
\mathbb{E}\left[d_{\gamma}\left(\eta(t), \eta^{\prime}(t)\right)\right] \leqslant \sum_{i=1}^{r-1} \mathbb{E}\left[d_{\gamma}\left(\zeta_{i}(t), \zeta_{i+1}(t)\right],\right.
$$


where we call $\zeta_{1}, \ldots, \zeta_{r}$ the minimizing path in (3.12) and $\zeta_{1}(t), \ldots, \zeta_{r}(t)$ the corresponding trajectory. In particular, subtracting $d_{\gamma}\left(\eta, \eta^{\prime}\right)=\sum_{i=1}^{r-1} d_{\gamma}\left(\zeta_{i}, \zeta_{i+1}\right)$, dividing by $t$ and letting $t \downarrow 0$ we obtain

$$
\left[\widetilde{\mathcal{G}} d_{\gamma}\right]\left(\eta, \eta^{\prime}\right) \leqslant \sum_{i=1}^{r-1}\left[\widetilde{\mathcal{G}} d_{\gamma}\right]\left(\zeta_{i}, \zeta_{i+1}\right) .
$$

Since each term in the r.h.s. above is of the form (3.6) with $d_{0}\left(\zeta_{i}, \zeta_{i+1}\right)=1$ we have that the assumptions imply

$$
\left[\widetilde{\mathcal{G}} d_{\gamma}\right]\left(\eta, \eta^{\prime}\right) \leqslant-d_{\gamma}\left(\eta, \eta^{\prime}\right)
$$

for arbitrary initial conditions. Therefore $\varphi(t):=\mathbb{E}\left[d_{\gamma}\left(\eta(t), \eta^{\prime}(t)\right)\right]$ satisfies $\frac{d}{d t} \varphi(t) \leqslant-$ $\varphi(t)$, which implies the claim since $\varphi(0) \leq \max _{\eta, \eta^{\prime}} d_{\gamma}\left(\eta, \eta^{\prime}\right) \leq k N(h, n)$.

3.2. A dynamics with full polymer moves. The next ingredient which enters the proof of Theorem 1.1 is a dynamics where each move consists in updating $(2 s+1)$ whole polymers, $s \in \mathbb{N}$. As usual, we let $\xi, \sigma \in \Omega_{1, n}^{h}$ (with $\sigma \leqslant \xi$ ) and $\mu^{\xi, \sigma}(\cdot)$ denotes the law on $E_{\xi, \sigma}$ for $k$ polymers with floor and ceiling $\sigma, \xi$, as defined in (1.3). To each $1 \leqslant j \leqslant k$ is assigned an independent Poisson clock of mean 1. When the clock labeled $j$ rings, we update the polymers $\eta^{(u)}$ with index $\max (j-s, 1) \leqslant u \leqslant \min (j+s, k)$, sampling the new configuration according to the law

$$
\hat{\nu}_{j, s}(\cdot):=\mu^{\xi, \sigma}\left(\cdot \mid \eta^{(\max (j-s-1,0))}, \eta^{(\min (j+s+1, k+1))}\right),
$$

with the convention that $\eta^{(0)}:=\xi$ and $\eta^{(k+1)}:=\sigma$. Call $\mathcal{M}$ the generator of this dynamics.

Proposition 3.5. For every $\alpha>0$ there exists $s:=s(\alpha) \in \mathbb{N}$ such that, uniformly in $n, k,|h| \leqslant n$ and on $\sigma \leqslant \xi$, one has

$$
\operatorname{gap}(\mathcal{M}) \geqslant 1 .
$$

Proof. The general structure of the proof is similar to that of Proposition 3.1, but the coupling argument is rather different. Given $\rho>0$, we define the distance function $D_{\rho}(\cdot, \cdot)$ by setting for every $\eta, \eta^{\prime}$,

$$
D_{\rho}\left(\eta, \eta^{\prime}\right):=\sum_{j=1}^{k} e^{-j \rho} \sum_{x=1}^{n-1} \frac{\left|\eta_{x}^{(j)}-\eta_{x}^{\prime(j)}\right|}{2}
$$

In analogy with (3.12), one checks that

$$
D_{\rho}\left(\eta, \eta^{\prime}\right)=\min _{\omega \in \Omega\left(\eta, \eta^{\prime}\right)} \sum_{i=1}^{r-1} D_{\rho}\left(\omega_{i}, \omega_{i+1}\right),
$$

where in this case one requires that $D_{0}\left(\omega_{i}, \omega_{i+1}\right)=1$ for $i<r$.

Given two initial conditions $\left(\eta, \eta^{\prime}\right)$, let $\left(\eta(t), \eta^{\prime}(t)\right)$ be the corresponding evolutions. As in the proof of Proposition 3.1, cf. Lemma 3.4, to prove (3.14), it is then sufficient 
to prove that for every $\alpha>0$ there exists a choice of $s \in \mathbb{N}, \rho>0$ and a coupling $\mathbb{P}$ of $\left(\eta(t), \eta^{\prime}(t)\right)$ such that

$$
\left[\widetilde{\mathcal{M}} D_{\rho}\right]\left(\eta, \eta^{\prime}\right):=\left.\frac{d}{d t} \mathbb{E}\left(D_{\rho}\left(\eta(t), \eta^{\prime}(t)\right)\right)\right|_{t=0} \leqslant-D_{\rho}\left(\eta, \eta^{\prime}\right)
$$

whenever $D_{0}\left(\eta, \eta^{\prime}\right)=1$.

Let $\left(\bar{\eta}, \bar{\eta}^{\prime}\right)$ satisfy the latter condition, with the single discrepancy consisting in $\bar{\eta}_{x}^{(i)}=$ $\bar{\eta}_{x}^{\prime(i)}+2$. In analogy with (3.7), we write the generator of the coupled dynamics as

$$
\widetilde{\mathcal{M}}:=\sum_{j=1}^{k}\left(\hat{E}_{j, s}-1\right),
$$

where $\hat{E}_{j, s}$ is a coupling (to be specified) of $\hat{\nu}_{j, s}$ for the two configurations. For all $j$ such that $i-s \leqslant j \leqslant i+s$ we can choose the coupling such that $\left[\hat{E}_{j, s} D_{\rho}\right]\left(\bar{\eta}, \bar{\eta}^{\prime}\right)=0$. Moreover, if $j \in\{1, \ldots, k\} \backslash\{i-s-1, \ldots, i+s+1\}$ we can choose the coupling such that $\left[\hat{E}_{j, s} D_{\rho}\right]\left(\bar{\eta}, \bar{\eta}^{\prime}\right)=D_{\rho}\left(\bar{\eta}, \bar{\eta}^{\prime}\right)$. One has therefore

$$
\begin{aligned}
& {\left[\widetilde{\mathcal{M}} D_{\rho}\right]\left(\bar{\eta}, \bar{\eta}^{\prime}\right)} \\
& \quad \leqslant-s D_{\rho}\left(\bar{\eta}, \bar{\eta}^{\prime}\right)+\left[\left(\hat{E}_{i+s+1, s}-1\right) D_{\rho}\right]\left(\bar{\eta}, \bar{\eta}^{\prime}\right)+\left[\left(\hat{E}_{i-s-1, s}-1\right) D_{\rho}\right]\left(\bar{\eta}, \bar{\eta}^{\prime}\right) .
\end{aligned}
$$

It is clear that the last two terms may be non-negative, and that they vanish if $i \geqslant k-s$ or $i \leqslant s+1$, respectively.

Let us analyze first the easier case of $\hat{E}_{i+s+1, s}$ in which case, it is worth recalling, we are updating polymers labeled $i+1, \ldots, i+2 s+1$. Since $\bar{\eta}^{(i)} \geqslant \bar{\eta}^{(i)}$ while $\bar{\eta}^{(i+2 s+2)}=$ $\bar{\eta}^{\prime(i+2 s+2)}$, by monotonicity there exists a coupling $\hat{E}_{i+s+1, s}$ such that one has $\eta^{(j)} \geqslant \eta^{(j)}$ for every $i<j<i+2 s+2$. Moreover, since $\bar{\eta}^{(i)}$ and $\bar{\eta}^{\prime(i)}$ differ only at $x$, we can choose $\hat{E}_{i+s+1, s}$ such that $\eta_{y}^{(j)}=\eta_{y}^{(j)}$ for every $i<j<i+2 s+2$ and $y$ outside the interval $\left\{a_{-}, \ldots, a_{+}\right\}$, where $a_{+}:=\inf \left\{y>x: \eta_{y}^{\prime(i+2 s+1)}=\bar{\eta}_{y}^{(i)}\right\}$ and $a_{-}:=\sup \{y<x$ : $\left.\eta_{y}^{(i+2 s+1)}=\bar{\eta}_{y}^{(i)}\right\}$. As a consequence, going back to the definition of $D_{\rho}(\cdot, \cdot)$,

$$
\left[\hat{E}_{i+s+1, s} D_{\rho}\right]\left(\bar{\eta}, \bar{\eta}^{\prime}\right) \leqslant \sum_{j=i+1}^{\infty} e^{-j \rho_{\nu_{i+s+1, s}}}\left(\left(a_{+}-a_{-}\right)^{2}\right) .
$$

Thanks to (2.9) there exists $c_{3}(\alpha)>0$ such that for every $u>0$

$$
\hat{\nu}_{i+s+1, s}\left(a_{+}-a_{-}=u\right) \leqslant e^{-c_{3}(\alpha) u} .
$$

From this one deduces immediately that there exists $c_{4}(\alpha, \rho)<\infty$ such that

$$
\left[\hat{E}_{i+s+1, s} D_{\rho}\right]\left(\bar{\eta}, \bar{\eta}^{\prime}\right) \leqslant c_{4}(\alpha, \rho) D_{\rho}\left(\bar{\eta}, \bar{\eta}^{\prime}\right) .
$$

Finally we deal with $\hat{E}_{i-s-1, s}$. We have from (3.15)

$$
\left[\hat{E}_{i-s-1, s} D_{\rho}\right]\left(\bar{\eta}, \bar{\eta}^{\prime}\right)=\frac{1}{2} \sum_{j=i-2 s-1}^{i-1} e^{-\rho j} \hat{E}_{i-s-1, s}\left(\sum_{y=1}^{n-1}\left|\eta_{y}^{(j)}-\eta_{y}^{\prime(j)}\right|\right) .
$$


Again, we can choose the coupling such that $\eta^{(j)} \leqslant \eta^{(j)}$ and $\eta_{y}^{(j)}=\eta_{y}^{\prime(j)}$ for $y \notin$ $\left\{b_{-}, \ldots, b_{+}\right\}$, where $b_{-}=\sup \left\{y<x: \eta_{y}^{\prime(i-1)}=\bar{\eta}_{y}^{(i-2 s-2)}\right\}$, and similarly for $b_{+}$. In analogy with (3.21) one has

$$
\hat{\nu}_{i-s-1, s}\left(b_{+}-b_{-}=u\right) \leqslant e^{-c_{3}(\alpha) u} .
$$

Then,

$$
\sum_{y=1}^{n-1}\left|\eta_{y}^{(j)}-\eta_{y}^{\prime(j)}\right| \leqslant\left|b_{+}-b_{-}\right| \times\left|\left\{b_{-}<y<b_{+}: \eta_{y}^{\prime(j)} \neq \bar{\eta}_{y}^{(i-2 s-2)}\right\}\right| .
$$

Using (3.25), (2.8) and (3.24),

$$
\begin{aligned}
\hat{E}_{i-s-1, s}\left(\sum_{y=1}^{n-1}\left|\eta_{y}^{(j)}-\eta_{y}^{\prime(j)}\right|\right) & =\hat{E}_{i-s-1, s}\left[\hat{E}_{i-s-1, s}\left(\sum_{y=1}^{n-1}\left|\eta_{y}^{(j)}-\eta_{y}^{\prime(j)}\right|\right) \mid b_{-}, b_{+}\right] \\
& \leqslant \hat{\nu}_{i-s-1, s}\left[\left(b_{+}-b_{-}\right)^{2}\right] e^{-c_{4}(\alpha)(i-j)} \leqslant c_{5}(\alpha) e^{-c_{4}(\alpha)(i-j)} .
\end{aligned}
$$

Therefore, going back to (3.23),

$$
\left[\hat{E}_{i-s-1, s} D_{\rho}\right]\left(\bar{\eta}, \bar{\eta}^{\prime}\right) \leqslant c_{5}(\alpha) D_{\rho}\left(\bar{\eta}, \bar{\eta}^{\prime}\right) \sum_{r=1}^{\infty} e^{-\left(c_{4}(\alpha)-\rho\right) r}=c_{6}(\alpha) D_{\rho(\alpha)}\left(\bar{\eta}, \bar{\eta}^{\prime}\right)
$$

where we chose $\rho:=\rho(\alpha)=c_{4}(\alpha) / 2$. From (3.19), (3.22) and (3.26) one concludes that

$$
\left[\widetilde{\mathcal{M}} D_{\rho(\alpha)}\right]\left(\bar{\eta}, \bar{\eta}^{\prime}\right) \leqslant-\left(s-c_{7}(\alpha)\right) D_{\rho(\alpha)}\left(\bar{\eta}, \bar{\eta}^{\prime}\right) .
$$

At this point, it is sufficient to choose $s:=s(\alpha):=\left\lceil c_{7}(\alpha)\right\rceil+1$ to get (3.17).

3.3. An estimate for $k$ polymers with $r$ particles. The last ingredient we need for the proof of Theorem 1.1 is a rough estimate on the spectral gap for a system with $k$ polymers, each with $r$ particles; it is important that this bound is independent of the lengths $n_{1}, \ldots, n_{k}$ of each polymer. Consider a configuration $\eta \in E_{\xi, \sigma} \subset \Omega_{k, n}^{h}$ with $k$ polymers, each with $N$ particles. Let $\mathbf{x}=\mathbf{x}(\eta)$ denote the corresponding particle configuration. Fix $0 \leqslant j<m \leqslant N+1$ and consider the probability measures $\nu_{j, m}^{\mathbf{x}}$ defined in (3.1). If we freeze all particles labeled $i \leqslant j$ and $i \geqslant m$ we can perform the local-update dynamics defined in (1.9) for the $r:=m-j-1$ particles labeled $j+1, \ldots, m-1$. This process is clearly reversible with respect to $\nu_{j, m}^{\mathbf{x}}$. Its Dirichlet form is given by

$$
\mathcal{E}_{j, m}^{\mathbf{x}}(f)=\sum_{u=1}^{k} \sum_{x=x_{j}^{(u)}+1}^{x_{m}^{(u)}-1} \nu_{j, m}^{\mathbf{x}}\left[\operatorname{Var}_{u, x}(f)\right],
$$

where $\operatorname{Var}_{u, x}(f)$ has the same meaning as in (1.9). As usual, below we use the notation $\operatorname{Var}_{\nu}(f)=\nu\left(f^{2}\right)-\nu(f)^{2}$ for any probability measure $\nu$.

Lemma 3.6. For every $\alpha>0, k \in \mathbb{N}$ and $r \in \mathbb{N}$, there exists $c=c(\alpha, k, r)$ such that for any pair $j, m$ with $m-j-1=r$, for any choice of all other parameters and for any $f: \Omega_{k, n}^{h} \rightarrow \mathbb{R}$

$$
\mathcal{E}_{j, m}^{\mathbf{x}}(f) \geqslant c \operatorname{Var}_{\nu_{j, m}^{\mathbf{x}}}(f) \text {. }
$$


Proof. The only delicate point here is that the length of the portion of paths where the $r$ particles live is arbitrarily long and we need an estimate which does not depend on that. We introduce a further family of measures as follows. Let $\nu_{j, m}^{\mathbf{x}, u}$ denote the law of $\eta^{(u)}$ according to the measure $\nu_{j, m}^{\mathbf{x}}$ conditioned on the value of the paths $\eta^{(i)}, i \neq u$. If we let

$$
\mathcal{E}_{j, m}^{\mathbf{x}, u}(f)=\sum_{x=x_{j}^{(u)}+1}^{x_{m}^{(u)}-1} \nu_{j, m}^{\mathbf{x}, u}\left[\operatorname{Var}_{u, x}(f)\right]
$$

denote the corresponding Dirichlet form we know from the $k=1$ version of Theorem 1.1 (see the remark following the statement of Theorem 1.1) that

$$
\mathcal{E}_{j, m}^{\mathbf{x}, u}(f) \geqslant \delta(\alpha) \operatorname{Var}_{\nu_{j, m}^{\mathbf{x}, u}}(f),
$$

for some constant $\delta(\alpha)$ depending only on $\alpha$. Taking expectation w.r.t. $\nu_{j, m}^{\mathbf{x}}$, using $\nu_{j, m}^{\mathbf{x}}\left[\nu_{j, m}^{\mathbf{x}, u}(g)\right]=\nu_{j, m}^{\mathbf{x}}(g)$ for any function $g$, and summing over $u$ in (3.28), by definition of $\mathcal{E}_{j, m}^{\mathbf{x}}(f)$ we have

$$
\mathcal{E}_{j, m}^{\mathbf{x}}(f) \geqslant \delta(\alpha) \sum_{u=1}^{k} \nu_{j, m}^{\mathbf{x}}\left[\operatorname{Var}_{\nu_{j, m}^{\mathbf{x}, u}}(f)\right] .
$$

Therefore it remains to prove that for some $c=c(\alpha, r, k)>0$ one has

$$
\sum_{u=1}^{k} \nu_{j, m}^{\mathbf{x}}\left[\operatorname{Var}_{\nu_{j, m}^{\mathbf{x}, u}}(f)\right] \geqslant c \operatorname{Var}_{\nu_{j, m}^{\mathbf{x}}}(f),
$$

for all functions $f$. To prove this estimate we observe that the left-hand side of (3.29) coincides with the Dirichlet form of the Markov chain described as follows. Attach independent rate 1 Poisson clocks to the labels $u=1, \ldots, k$. When a label $u$ rings update the whole path between $x_{j}^{(u)}+1$ and $x_{m}^{(u)}-1$ according to the distribution $\nu_{j, m}^{\mathbf{x}, u}$ (that is, freeze all other polymers and update polymer $u$ with a sample from $\nu_{j, m}^{\mathbf{x}, u}$ ).

Thus, the following rough coupling argument will suffice for the proof of (3.29). Namely, consider the Markov chain started in the minimal configuration (i.e. each of the $k$ polymers starts in the minimal path compatible with the particles $x_{j}^{(u)}$ and $\left.x_{m}^{(u)}\right)$. Let $E_{t}$ denote the event that up to time $t$ the Markov chain has never visited the maximal allowed configuration. It is not hard to prove a bound of the form $\mathbb{P}\left(E_{t}\right) \leqslant c^{-1} e^{-c t}$ for some $c=c(\alpha, r, k)>0$. This, in turn, implies the desired spectral gap estimate (3.29) using monotonicity and the bound (3.4).

To prove $\mathbb{P}\left(E_{t}\right) \leqslant c^{-1} e^{-c t}$ we may reason as follows. Consider the event $F_{s}$ that in the time interval $[s, s+1)$ the following sequence of $k$ successive updatings appears: for $i=1, \ldots, k$ the $i$-th update is for polymer $u=i$ and the configuration sampled from $\nu_{j, m}^{\mathbf{x}, u}$ is the maximal allowed path for the $i$-th polymer given the current configuration. Since there are $r$ particles, an application of Lemma 2.2 shows that $\mathbb{P}\left(F_{s}\right) \geqslant p$ where $p=p(\alpha, r, k)>0$ depends neither the lengths $n_{u}=x_{m}^{(u)}-x_{j}^{(u)}$ of the polymers, nor on the configuration of $\eta$ at time $s$. By construction, on the event $F_{s}$ we know that the Markov chain visited the maximal allowed configuration at least once in the time interval $[s, s+1)$. Therefore the event $E_{t}$ implies that none of the events $F_{s}$ occurred 
for $s=0, \ldots,\lfloor t-1\rfloor$. This implies $\mathbb{P}\left(E_{t}\right) \leqslant(1-p)^{t-1} \leqslant(1-p)^{-1} e^{-p t}$. The proof of Lemma 3.6 is complete.

3.4. Putting everything together: proof of Theorem 1.1. Once Proposition 3.1, Proposition 3.5 and Lemma 3.6 are established, the proof of Theorem 1.1 is obtained through a chain of comparison inequalities. Indeed, Proposition 3.5 may be restated as

$$
\operatorname{Var}(f) \leqslant \sum_{j=1}^{k} \mu\left[\operatorname{Var}_{\hat{\nu}_{j, s}}(f)\right]
$$

where $\operatorname{Var}(f)$ is the variance w.r.t. $\mu:=\mu^{\xi, \sigma}$ and $\hat{\nu}_{j, s}$ is the conditional probability measure defined in (3.13). Here $s=s(\alpha)$ is a fixed parameter $(2 s+1$ is the number of polymers to be updated at each step in the process with generator $\mathcal{M}$ appearing in Proposition 3.5 they are the polymers labeled $u$, with $\max (j-s-1,1) \leq u \leq$ $\min (j+s+1, k))$. For each $j$ in (3.30) we apply the estimate of Proposition 3.1 with the number of polymers equal to $k=s$. This yields

$$
\operatorname{Var}_{\hat{\nu}_{j, s}}(f) \leqslant \sum_{i=1}^{N} \hat{\nu}_{j, s}\left[\operatorname{Var}_{\rho_{i, \ell}^{\mathbf{x}}}(f)\right]
$$

where $\rho_{i, \ell}^{\mathbf{x}}$ is the conditional probability measure appearing in (3.3) and $\ell=\ell(\alpha)$ is a fixed parameter $(2 \ell+1$ is the size of the block of particles to be updated at each step in the process with generator $\mathcal{G}$ appearing in Proposition 3.1). Finally, recalling the definition $\rho_{i, \ell}^{\mathbf{x}}=\nu_{i-\ell-1, i+\ell+1}^{\mathbf{x}}$ (cf. (3.1)) and applying the bound in Lemma 3.6 (with $r=2 \ell+1$ and $k=s$ ) we know that for every $i$ in (3.31) we have the estimate for all $\mathbf{x}=\mathbf{x}(\eta)$ :

$$
\operatorname{Var}_{\rho_{i, \ell}^{\mathbf{x}}}(f) \leqslant c^{-1} \sum_{u=j-s-1}^{j+s+1} \sum_{x=x_{i-\ell-1}^{(u)}+1}^{x_{i+\ell+1}^{(u)}-1} \rho_{i, \ell}^{\mathbf{x}}\left[\operatorname{Var}_{u, x}(f)\right]
$$

Note that the constant $c=c(\alpha, s, \ell)$ depends only on $\alpha$ here. Also, note that since there are at most $2 \ell+1$ blocks of particles covering a given position $x$ in the path we obtain

$$
\sum_{i=1}^{N} \hat{\nu}_{j, s}\left[\operatorname{Var}_{\rho_{i, \ell}^{\mathbf{x}}}(f)\right] \leqslant c^{-1}(2 \ell+1) \sum_{u=j-s-1}^{j+s+1} \sum_{x=1}^{n-1} \hat{\nu}_{j, s}\left[\operatorname{Var}_{u, x}(f)\right]
$$

where we use the property of conditional expectation $\hat{\nu}_{j, s}\left[\rho_{i, \ell}^{\mathbf{x}}(g)\right]=\hat{\nu}_{j, s}(g)$ valid for any function $g$. 
Putting everything together and using $\mu\left[\hat{\nu}_{j, s}(g)\right]=\mu(g)$ for any $g$, from (3.30)-(3.33) we see that

$$
\begin{aligned}
\operatorname{Var}(f) & \leqslant c^{-1}(2 \ell+1) \sum_{j=1}^{k} \sum_{u=j-s-1}^{j+s+1} \sum_{x=1}^{n-1} \mu\left[\operatorname{Var}_{u, x}(f)\right] \\
& \leqslant c^{-1}(2 \ell+1)(2 s+1) \sum_{i=1}^{k} \sum_{x=1}^{n-1} \mu\left[\operatorname{Var}_{i, x}(f)\right] \\
& =c^{-1}(2 \ell+1)(2 s+1) \mathcal{E}(f) .
\end{aligned}
$$

This shows that $\operatorname{gap}(\alpha) \geqslant c^{\prime}(\alpha)$, with $c^{\prime}(\alpha)=c(2 \ell+1)^{-1}(2 s+1)^{-1}$, which completes the proof of Theorem 1.1.

\section{Proof of Theorem 1.2}

For clarity of exposition we give the proof first of all in the simpler case where the ceiling $\xi$ is the maximal configuration, $\wedge$, in $\Omega_{1, n}^{h}$. Later, in Section 4.2 we sketch the modifications which are needed in the general situation.

4.1. The case of maximal ceiling. By monotonicity and the definition (1.11) of mixing time, it is clear that a sufficient condition for $T_{\text {mix }} \leqslant T$ is that the first (random) time when the dynamics started from the minimal configuration, $\left\{\eta^{(j)}=\sigma, j=1, \ldots, k\right\}$, passes through the maximal configuration, $\left\{\eta^{(j)}=\xi, j=1, \ldots, k\right\}$, is smaller than $T$ with probability at least $1-1 /(2 e)$. Again by monotonicity, it is easy to convince oneself that this random time does not decrease if one replaces $h$ with zero, $n$ with $2 M:=2 \max (n, k), k$ with $M$ and $\sigma$ with $\vee$.

Therefore, to prove Theorem 1.2 (in the case of maximal ceiling) it is sufficient to prove the following. Let $h=0, n=2 M, k=M, \xi=\wedge, \sigma=\vee$, cf. (1.7), (1.8); start the dynamics from the minimal configuration $\eta_{-}:=\left\{\eta^{(j)}=\vee\right.$ for all $\left.j=1, \ldots, M\right\}$, and call $t(M)$ the first time when the maximal configuration $\eta^{+}:=\left\{\eta^{(j)}=\wedge\right.$ for all $j=1, \ldots, M\}$ is reached. Then,

Theorem 4.1. There exists $C(\alpha)>0$ such that with probability larger than $1-1 /(2 e)$

$$
t(M) \leqslant C(\alpha) M(\log M)^{6} .
$$

A notational convention When in the following we say that an event occurs "with large probability" (w.l.p.), we mean to say that the probability of the complementary event goes to zero for $M \rightarrow \infty$ faster than any inverse power of $M$. Since, as will be clear, we have to exclude only polynomially many (in $M$ ) events which w.l.p. do not occur, by the union bound we have that the occurrence of at least one of these events still goes to zero for $M \rightarrow \infty$. For simplicity of exposition, and when there is no risk of confusion, we will often just pretend that an event which occurs w.l.p., occurs deterministically. 
4.1.1. From polymer configurations to subsets of the cube. The proof of Theorem 4.1 becomes more intuitive if one interprets a configuration $\eta \in \Omega_{M, 2 M}^{0}$ as a subset $s$ of the cube $\mathcal{C}_{M}:=[0, M]^{3} \subset \mathbb{R}^{3}$. This mapping is just another way to see the mapping between polymer configurations $\eta$ and boxed plane partitions introduced in Section 1.1.1. To define precisely this correspondence, divide first of all $\mathcal{C}_{M}$ into $M^{3}$ elementary cubes of unit side, which we label with the (integer) coordinates $r=\left(r_{1}, r_{2}, r_{3}\right)$ of their point of smallest $L^{1}$ norm (observe that $0 \leqslant r_{i}<M$ ). Then, to a given $\eta \in \Omega_{M, 2 M}^{0}$ we associate $s=s(\eta) \subset \mathcal{C}_{M}$, a union of elementary cubes, by establishing that the elementary cube labeled $r$ belongs to $s$ if and only if (cf. Figure 5)

$$
\eta_{M-r_{1}+r_{2}}^{\left(M-r_{3}\right)}<M-r_{1}-r_{2} .
$$
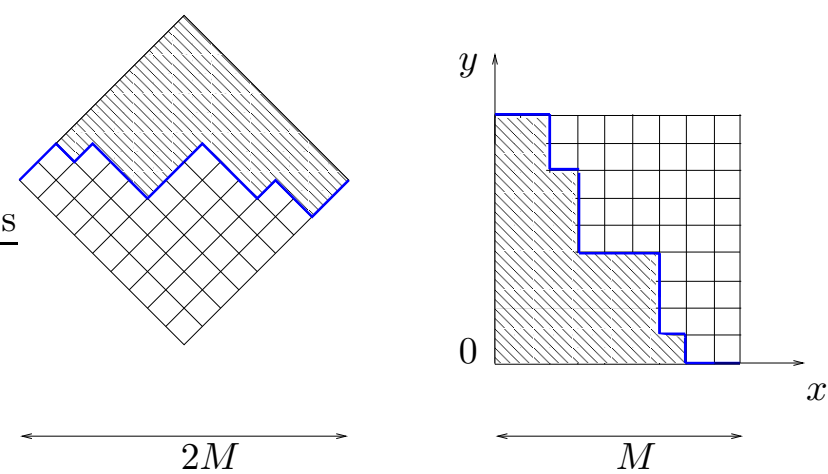

Figure 5. A graphical construction of the set $s(\eta)$. Given $\eta \in \Omega_{M, 2 M}^{0}$, in order to obtain $s(\eta)$ do the following, for $r_{3}=0, \ldots,(M-1)$ : a) draw the configuration of $\eta^{\left(M-r_{3}\right)}$ (thick line in the left drawing); b) rotate the picture by $3 \pi / 4$ anti-clockwise and shrink it by a scale factor $1 / \sqrt{2}$. The shaded region coincides with the horizontal section of $s(\eta)$ at height $r_{3}<h \leqslant r_{3}+1$. It is obvious from this construction that the subset $s(\eta)$ thus obtained is a monotone subset of the cube $\mathcal{C}_{M}$.

It is easy to check that:

- if $\eta \leqslant \eta^{\prime}$ then $s^{\prime} \subset s$ (note that the inequality is reversed!)

- the maximal configuration $\eta^{+}$defined above corresponds to the empty subset of the cube: $s^{-}=\emptyset$. Conversely, the minimal polymer configuration $\eta^{-}$corresponds to the maximal subset $s^{+}=\mathcal{C}_{M}$, i.e., the full cube. We will sometimes refer to $s^{-}$as to the ground state, for obvious reasons.

- for every $\eta \in \Omega_{M, 2 M}^{0}, s(\eta)$ is a monotone subset of $\mathcal{C}_{M}$, i.e., if the elementary cube labeled $r$ belongs to $s$, then so do also all the elementary cubes $r^{\prime}$ such that $r_{1}^{\prime} \leqslant r_{1}, r_{2}^{\prime} \leqslant r_{2}, r_{3}^{\prime} \leqslant r_{3}$.

The equilibrium measure can be described in terms of $s$ by:

$$
\mu^{\wedge, \vee}(s)=\frac{e^{-2 \alpha|s|}}{\hat{Z}_{\wedge, \vee}}
$$


where $|s|$ denotes the number of elementary cubes contained in $s$, i.e., its volume.

The dynamics of Section 1.2 can also be explicitly described in terms of $s$. Here, let us simply remark that the elementary moves of the Markov Chain consist in adding or removing a single elementary cube, with the constraint that $s$ remains a monotone subset of $\mathcal{C}_{M}$ after the update. Observe also that, under the $\eta \leftrightarrow s$ correspondence, the upward drift felt by the polymers $\eta^{(j)}$ during the dynamics translates into the fact that the upper boundary of $s$ feels a drift in the direction $(-1,-1,-1)$.

4.1.2. Proof of Theorem 4.1. To avoid a plethora of $\lfloor\cdot\rfloor$, we assume that $(\log M)^{2} \in 2 \mathbb{N}$ and that $K:=\left(M /(\log M)^{2}\right) \in \mathbb{N}$. Divide $\mathcal{C}_{M}$ into sub-cubes $B_{v}$ (called blocks from now on) of side $(\log M)^{2}$, indexed by $v=\left(v_{1}, v_{2}, v_{3}\right)$ with $0 \leqslant v_{i}<K$, and such that the point of $B_{v}$ with minimal $L^{1}$ norm is $(\log M)^{2} v$ (of course, $v_{i}$ are integers). Given $v$, we will also define $B_{v}^{+}, B_{v}^{-}$to be the half-blocks obtained cutting $B_{v}$ horizontally into two equal parts ( $B_{v}^{-}$will denote the bottom one). Call $s_{t}$ the configuration at time $t$, which starts from the completely full configuration $s^{+}$. The idea is to consider an easier dynamics $\hat{s}_{t}$ such that $s_{t} \subset \hat{s}_{t}$ almost surely and, calling $\hat{t}(M)$ the first time $\hat{s}_{t}$ reaches the empty configuration $s^{-}$, to show that $\hat{t}(M)$ satisfies (4.1) with probability at least $1-1 /(2 e)$.

Let

$$
\tau:=\tau(M):=C(\alpha)(\log M)^{8} / 7
$$

where $C(\alpha)$ is the same as in (4.1). We define now two deterministic sets $S_{t}^{ \pm} \subset$ $\mathcal{C}_{M}$ which (roughly speaking) coincide with $s^{+}$at $t=0$ and get empty in a time $C(\alpha) M(\log M)^{6}$, and such that $\hat{s}_{t}$ satisfies $S_{t}^{-} \subset \hat{s}_{t} \subset S_{t}^{+}$, w.l.p. and for all $t \leqslant M^{2}$.

$\left\{S_{t}^{-}\right\}_{t} \geqslant 0$, is defined as follows (see also Figure 6 for a graphical definition):

- $S_{0}^{-}=\mathcal{C}_{M}$

- if $i \in \mathbb{N}$ and $(i-1) \tau<t \leqslant i \tau$, then $S_{t}^{-}$contains all and only the blocks $B_{v}$ such that $v$ satisfies

$$
v_{3}+2\left(v_{1}+v_{2}\right) \leqslant 5(K-1)-i
$$

On the other hand, letting for ease of notation

$$
V_{t}^{ \pm}:=\mathcal{C}_{M} \backslash S_{t}^{ \pm}
$$

$\left\{S_{t}^{+}\right\}_{t \geqslant 0}$ is defined as follows (see also the caption of Fig. [6):

- $S_{t}^{-} \subset S_{t}^{+} \subset \mathcal{C}_{M}$

- If $B_{v} \in V_{\tau\lfloor t / \tau\rfloor}^{-}$and $B_{v-e_{i}} \in V_{\tau\lfloor t / \tau\rfloor}^{-}$for at least one choice of $i=1,2,3$, then $B_{v} \in V_{t}^{+}\left(e_{i}\right.$ are the canonical base vectors of $\mathbb{Z}^{3}$, and $\lfloor x\rfloor:=\max \{n \in \mathbb{Z}$ : $n \leqslant x\})$.

- If $B_{v} \in V_{\tau\lfloor t / \tau\rfloor}^{-}$but there is no $i=1,2,3$ such that $B_{v-e_{i}} \in V_{\tau\lfloor t / \tau\rfloor}^{-}$then $B_{v}^{+} \in V_{t}^{+}$ but $B_{v}^{-} \in S_{t}^{+}$.

The following properties of $S_{t}^{ \pm}$are immediately checked:

- $S_{t}^{-}$is the union of blocks $B_{v}$, while $S_{t}^{+}$is the union of blocks and of half-blocks $B_{v}^{-}$

- $S^{-}$is left-continuous, non-increasing, constant in the time intervals $((i-1) \tau, i \tau]$

- $S^{+}$is right-continuous, non-increasing and constant on intervals $[(i-1) \tau, i \tau)$ 
- $S_{t}^{ \pm}$are monotone subsets of $\mathcal{C}_{M}$ for every $t$.

- For $t \geqslant(6 / 7) C(\alpha) M(\log M)^{6}=6 K \tau, S_{t}^{-}$is empty and $S_{t}^{+}$contains only $B_{(0,0,0)}^{-}$ $\left(K:=M /(\log M)^{2}\right.$ was defined at the beginning of this section).

As an example, if $0<t \leqslant \tau$ then $S_{t}^{-}=\mathcal{C}_{M} \backslash B_{(K-1, K-1, K-1)}$, while if $2 \tau<t \leqslant 3 \tau$ then

$$
S_{t}^{-}=\mathcal{C}_{M} \backslash\left(B_{(K-2, K-1, K-1)} \cup B_{(K-1, K-2, K-1)} \cup_{i=1}^{3} B_{(K-1, K-1, K-i)}\right) .
$$

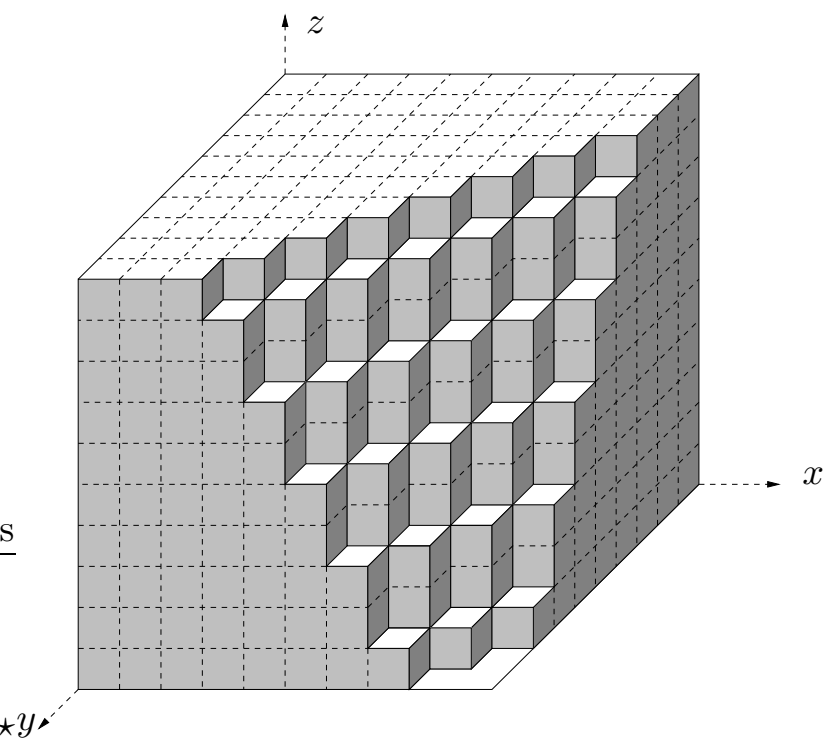

Figure 6 . The set $S_{t}^{-}$for $14 \tau<t \leqslant 15 \tau$. Small cubes denote blocks of side $(\log M)^{2}$, and there are $K=M /(\log M)^{2}(=10)$ of them along each side. At time intervals of $2 \tau$, a new diagonal set of columns with $v_{1}+v_{2}=$ const starts to move downwards: from then on, it moves one block down each time interval $\tau$. Once a column is empty, it stays empty forever. Roughly speaking, the set $S_{t}^{-}$contains all the blocks which are below a plane perpendicular to the vector $(2,2,1)$ and which moves at constant speed, of order $(\log M)^{-6}$, in the direction $(-2,-2,-1)$. The set $S_{t}^{+}$for $i \tau \leqslant t<(i+1) \tau$ can be obtained simply by taking $S_{t}^{-}$ for some $(i-1) \tau<t \leqslant i \tau$ and adding a half-blocks $B_{v}^{-}$on top of each incomplete but not empty column, and also to each empty column which is adjacent to a non-empty one $\left(S_{t}^{+}\right.$is not drawn in the picture).

We define the auxiliary dynamics $\hat{s}_{t}$ by establishing that it has the same law as $s_{t}$ conditioned on the event that $S_{t}^{-} \subset s_{t}$ for every $t \geqslant 0$. Remark that by monotonicity we can couple $s_{t}$ and $\hat{s}_{t}$ in such a way that $s_{t} \subset \hat{s}_{t}$ for every $t \geqslant 0$; also, remark that once $S_{t}^{-}$is empty there is no more constraint on the dynamics (which does not mean that $s_{t}=\hat{s}_{t}$ after that time!).

The basic point is the following: 
Theorem 4.2. W.l.p., for every $0 \leqslant t \leqslant M^{2}$ one has

$$
S_{t}^{-} \subset \hat{s}_{t} \subset S_{t}^{+} \text {. }
$$

Of course, the lower bound is trivial by the very definition of $\hat{s}_{t}$ (and holds not only w.l.p. but with probability one). Let us show that Theorem 4.2 implies (4.1). We know that for all times $(6 / 7) C(\alpha) M(\log M)^{6} \leqslant t \leqslant M^{2}$ one has $\hat{s}_{t} \subset S_{t}^{+}=B_{(0,0,0)}^{-}$, which is a subset of a cube of side $(\log M)^{2}$. By Lemma 4.3 below, this means that, within time $(6 / 7) C(\alpha) M(\log M)^{6}+O\left((\log M)^{6}\right) \leqslant C(\alpha) M(\log M)^{6} \ll M^{2}, \hat{s}_{t}$ has reached the ground state at least once with probability at least $1-1 /(2 e)$, and (4.1) follows. Of course, $M^{2}$ could be replaced by $M^{1+\epsilon}$ for any $\epsilon>0$.

The first ingredient of the proof of Theorem 4.2 is the following lemma, which gives a rough upper bound on the mixing time in a cube of size $M$ :

Lemma 4.3. For every $\alpha>0$ there exists $C_{1}(\alpha)<\infty$ such that for every $M \in \mathbb{N}$

$$
T_{\text {mix }} \leqslant C_{1}(\alpha) M^{3} \text {. }
$$

Moreover, there exists $C_{2}(\alpha)>0$ such that for every $T>0$ the following is true:

(1) the probability that $t(M) \geqslant T M^{3}$ is smaller than $\exp \left(-C_{2}(\alpha) T\right)$.

(2) with probability at least $1-T e^{-C_{2}(\alpha) M}$, the volume of $s_{t}$ is at most $M / 10$ for all times $t(M) \leqslant t \leqslant T$.

Proof. We know that

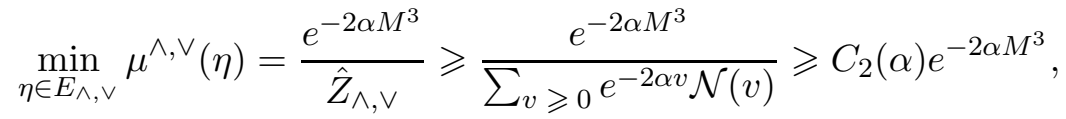

where $\mathcal{N}(v)$ was defined after formula (2.6) and is just the number of plane partitions of volume $v$. Then, it follows from (1.13) (and the fact that the gap is uniformly positive) that the mixing time is $O\left(M^{3}\right)$.

From (4.7) it is immediate to deduce (modulo redefining $C_{1}(\alpha)$ ) that the probability that $t(M) \leqslant C_{1}(\alpha) M^{3}$ is greater than some $\epsilon(\alpha)>0$. (Indeed, Lemma 2.1 implies that there exists a set $A$ of configurations such that $\mu^{\wedge, \vee}(A)>1 / 2$ and such that all the configurations $s \in A$ can be reached via at most $m=m(\alpha)$ Markov Chain moves from the ground state $s^{-}$, for some $m(\alpha)<\infty$ independent of $M$ ). From this, one easily deduces that, for every $n \in \mathbb{N}$, the probability that $t(M)>n C_{1}(\alpha) M^{3}$ is smaller than $(1-\epsilon(\alpha))^{n}$, i.e., claim (1). Indeed, if the evolution has not passed through $s^{-}$before time $(n-1) C_{1}(\alpha) M^{3}$, just restart the dynamics from the maximal configuration $s^{+}$at $t=(n-1) C_{1}(\alpha) M^{3}$ : this can only make $t(M)$ larger, by monotonicity.

To prove statement (2), observe first of all that for all times $t>t(M)$ (or, more precisely, conditionally on $t(M)<t$ ) the distribution of $s_{t}$ is stochastically dominated by the equilibrium distribution $\mu^{\wedge, \vee}$. On the other hand, Lemma 2.1 tells us that

$$
\mu^{\wedge, \vee}(|s| \geqslant M / 10) \leqslant \exp \left(-c_{1}(\alpha) M / 10\right) .
$$

Secondly, the number of Markov Chain moves in the interval $[0, T]$ is a Poisson random variable $\zeta$ with average $T M^{2}$, and an elementary computation shows that for a Poisson random variable $\zeta_{\lambda}$ of parameter $\lambda$ one has

$$
\mathbb{P}\left(\zeta_{\lambda} \geqslant n\right) \leqslant e^{-n(\log (n / \lambda)-1)} .
$$


We have therefore, calling $t_{i}, i=1, \ldots, \zeta$ the random times when the updates occur,

$$
\begin{aligned}
\mathbb{P}\left(\exists t \in[t(M), T]:\left|s_{t}\right|>M / 10\right) \leqslant & e^{-M^{2} T} \\
& +\mathbb{P}\left(\zeta \leqslant 4 M^{2} T ; \exists i: t(M) \leqslant t_{i} \leqslant T,\left|s_{t_{i}}\right| \geqslant M / 10\right) \\
& \leqslant e^{-M^{2} T}+4 M^{2} T \exp \left(-c_{1}(\alpha) M / 10\right),
\end{aligned}
$$

where in the last inequality we used the union bound and (4.9).

Notational convention: in the rest of this section, we will use for simplicity of exposition expressions like "for all times larger than $t_{0}$ " to mean "for all times $t_{0} \leqslant t \leqslant M^{2}$ ".

For the next lemma we need some notations. Let $0 \leqslant j<2(K-1)$ and let $\mathcal{S}(j)$ be the set of configurations $s$ such that $B_{v} \subset s$ if $\left(v_{1}+v_{2}\right)<j$ and $B_{v} \cap s=\emptyset$ if $\left(v_{1}+v_{2}\right)>j+1$ or if $v_{1}+v_{2} \in\{j, j+1\}$ and $v_{3} \geqslant 2$. Let $s_{j}^{\max }$ be the maximal configuration in $\mathcal{S}(j)$, see Figure 7 (of course, both $\mathcal{S}(j)$ and $s_{j}^{\max }$ depend on $M$ ). We define a dynamics $\left\{s_{t}^{(j)}\right\}_{t} \geqslant 0$ by requiring that (in law) it equals our usual dynamics, with initial condition $s_{t=0}^{(j)}=s_{j}^{\max }$ and conditioned on the event that $s_{t}^{(j)} \in \mathcal{S}(j)$ for every $t \geqslant 0$.

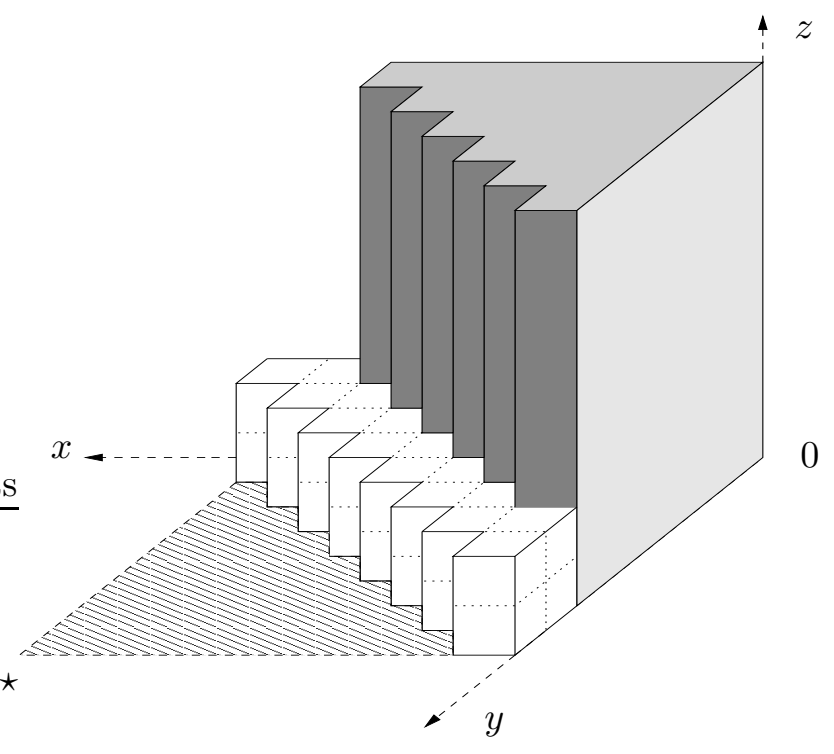

Figure 7. The maximal configuration $s_{j}^{\max }=: s_{t=0}^{(j)}$ in $\mathcal{S}(j)$. The dark region is the one which is constrained to remain completely full during the evolution of $s_{t}^{(j)}$, while the dashed one remains empty. The white region is the one which can evolve. Note that, for graphical convenience, the axes are drawn with orientations which differ from those of Fig. 6,

Lemma 4.4. W.l.p., the following holds for all times $t \geqslant \tau$. If $\left(v_{1}+v_{2}\right)=j$ and $v_{3}=1$, or if $\left(v_{1}+v_{2}\right)=j+1$, then $B_{v} \cap s_{t}^{(j)}=\emptyset$. If $\left(v_{1}+v_{2}\right)=j$ and $v_{3}=0, B_{v}^{+} \cap s_{t}^{(j)}=\emptyset$.

Proof of Lemma 4.4 As in the proof of Theorem 4.1, we introduce an auxiliary dynamics $\left\{\hat{s}_{t}^{(j)}\right\}_{t \geqslant 0}$ for which the claim is easier to prove, and such that almost surely 
$s_{t}^{(j)} \subset \hat{s}_{t}^{(j)}$ (so that the claim follows also for $\left.s_{t}^{(j)}\right)$. The auxiliary dynamics is defined simply by requiring that its law equals that of $\left\{s_{t}^{(j)}\right\}_{t} \geqslant 0$ conditioned on the event that, for all times $0 \leqslant t \leqslant \tau / 2, B_{v} \subset s_{t}^{(j)}$ if $\left(v_{1}+v_{2}\right)=j$ and $v_{1} \in 2 \mathbb{N}+1$. In other words, such blocks $B_{v}$ are frozen and remain completely full up to time $\tau / 2$. This implies that during this time interval the blocks which are not frozen evolve independently by groups of at most six, see Figure 8. Applying Lemma 4.3, one sees that at time $\tau / 2$

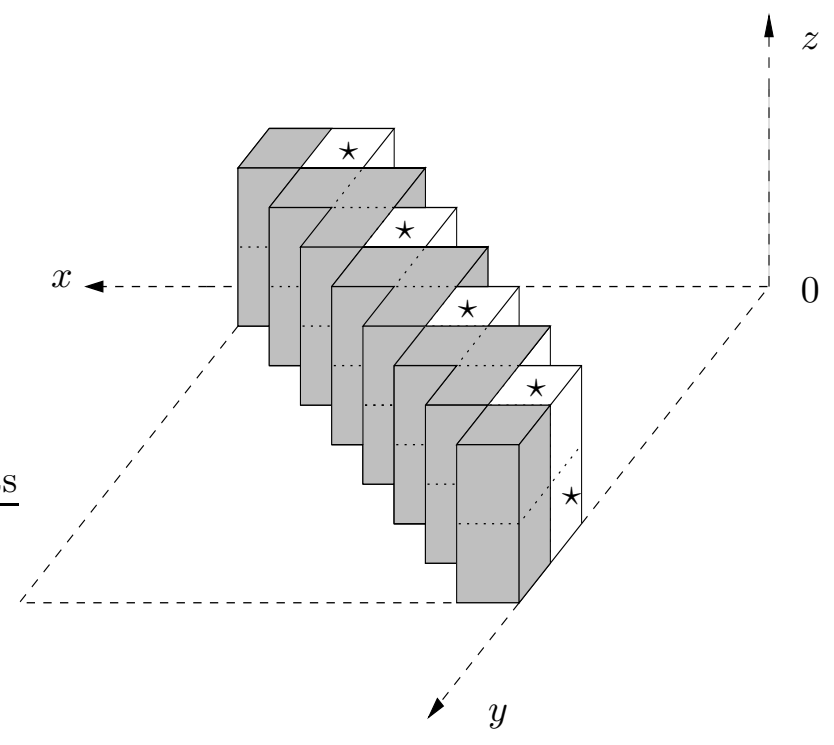

FiguRE 8. Here we drew only the region where $s_{t}^{(j)}$ is allowed to evolve (the white region in Fig. 7). Under the dynamics $\hat{s}_{t}^{(j)}$, blocks marked by $a \star$ are constrained to remain full up to time $\tau / 2$. As a consequence, up to that time the remaining blocks (shown in gray) evolve independently by groups of at most six.

the situation is the following (w.l.p.):

(1) $B_{v} \cap \hat{s}_{\tau / 2}^{(j)}=\emptyset$ if $\left(v_{1}+v_{2}\right)=j+1$ or $\left(v_{1}+v_{2}\right)=j, v_{1} \in 2 \mathbb{N}$ and $v_{3}=1$; the same remains true for all later times (of course here we are using the fact that, since $\tau=C(\alpha)(\log M)^{8} / 7$, the probability that $t\left((\log M)^{2}\right) \geqslant \tau / 2$ is $O\left(\exp \left(-C^{\prime}(\alpha)(\log M)^{2}\right)\right)$, cf. statement (1) of Lemma4.3, i.e., w.l.p. such event does not occur.)

(2) If $\left(v_{1}+v_{2}\right)=j, v_{1} \in 2 \mathbb{N}$ and $v_{3}=0$, the volume of $B_{v} \cap \hat{s}_{\tau / 2}^{(j)}$ is at most $(\log M)^{2} / 10$ (in particular, $B_{v}^{+} \cap \hat{s}_{\tau / 2}^{(j)}=\emptyset$ ); the same remains true for all later times. Here we are using statement (2) of Lemma 4.3, with $T=M^{2}$.

(3) all blocks marked by a star in Figure 8 are of course still completely full.

During the interval $(\tau / 2, \tau]$ the blocks marked by $\mathrm{a} \star$ in Fig. 8 are free to move. However, now the pairs of blocks $v_{1}+v_{2}=j, v_{1} \in 2 \mathbb{N}+1, v_{3} \in\{0,1\}$ evolve independently for $v_{1}$ different, thanks to point (1) above. Therefore, applying once more Lemma 4.3 and point (2) above, one sees that for $t \geqslant \tau$ one has $B_{v} \cap \hat{s}_{t}^{(j)}=\emptyset$ for 
$v_{1}+v_{2}=j, v_{1} \in 2 \mathbb{N}+1, v_{3}=1$, and $B_{v}^{+} \cap \hat{s}_{t}^{(j)}=\emptyset$ for $v_{1}+v_{2}=j, v_{1} \in 2 \mathbb{N}+1, v_{3}=0$. The claim is proven.

Proof of Theorem 4.2 It is sufficient to prove the following: for every $i \in \mathbb{N} \cup\{0\}$ such that $i \tau \leqslant M^{2}$, one has (w.l.p.)

$$
\hat{s}_{t} \subset S_{i \tau}^{+} \text {for every } t \geqslant i \tau \text {. }
$$

For $i=0$ the statement is trivial since $S_{0}^{+}=\mathcal{C}_{M}$.

Now we assume that the claim is true up to a certain $i$, and we show that it holds also for $i+1$. It is convenient to introduce the definition of column $C_{v_{1}, v_{2}}(H)$ with (integer) base coordinates $0 \leqslant v_{1}, v_{2}<K$ and height $0 \leqslant H \leqslant K$ : this is just a parallelepiped of height $(\log M)^{2} H$ and whose base is the square of side $(\log M)^{2}$ such that the base point of minimal $L^{1}$ norm has coordinates $\left((\log M)^{2} v_{1},(\log M)^{2} v_{2}\right)$. Note that, for each $t, S_{t}^{ \pm}$can be viewed as composed of $K^{2}$ such columns: in the case of $S_{t}^{-}$the heights $H_{t}^{-}\left(v_{1}, v_{2}\right)$ take values in $\{0,1, \ldots, K\}$ while in the case of $S_{t}^{+}$we call them $H_{t}^{+}\left(v_{1}, v_{2}\right)$ and they take values in $\{0,1 / 2,1, \ldots, K-1 / 2, K\}$. Note also that, by construction, $H_{t}^{ \pm}\left(v_{1}, v_{2}\right)$ depends on $\left(v_{1}, v_{2}\right)$ only through $v_{1}+v_{2}$ (see Fig. 6).

To complete the inductive proof, we need to prove that for all $\left(v_{1}, v_{2}\right)$

$$
\left[\hat{s}_{t} \cap C_{v_{1}, v_{2}}(K)\right] \subset C_{v_{1}, v_{2}}\left(H_{(i+1) \tau}^{+}\left(v_{1}, v_{2}\right)\right) \text { for every } t \geqslant(i+1) \tau .
$$

The following cases can occur (keep Fig. 6] in mind):

(1) $H_{(i+1) \tau}^{-}\left(v_{1}, v_{2}\right)=K$. In this case, (4.13) is obvious because also $H_{(i+1) \tau}^{+}\left(v_{1}, v_{2}\right)=$ $K$.

(2) $1<H_{(i+1) \tau}^{-}\left(v_{1}, v_{2}\right)<K$. In this case, we have that

$C_{v_{1}, v_{2}}\left(H_{(i+1) \tau}^{-}\left(v_{1}, v_{2}\right)\right) \subset\left[\hat{s}_{t} \cap C_{v_{1}, v_{2}}(K)\right] \subset C_{v_{1}, v_{2}}\left(H_{(i+1) \tau}^{-}\left(v_{1}, v_{2}\right)+2\right)$

for every $i \tau<t<(i+1) \tau$. The lower bound is trivial by the definition of the dynamics $\hat{s}_{t}$, while the upper bound follows from the inductive hypothesis (4.12) and from the fact that the definition of $S_{t}^{+}$implies that $0 \leqslant H_{t}^{+}\left(v_{1}, v_{2}\right)-$ $H_{t}^{-}\left(v_{1}, v_{2}\right)<2$. Since we want to prove (4.13), by monotonicity we can assume that all the columns labeled $\left(w_{1}, w_{2}\right)$ with $w_{1}+w_{2}<v_{1}+v_{2}$ are completely full during the time interval $i \tau<t<(i+1) \tau$. But then, as we shall argue in a moment, in the time interval $(i \tau,(i+1) \tau)$ the column $\hat{s}_{t} \cap C_{v_{1}, v_{2}}(K)$ evolves independently of all the others, and an application of Lemma 4.3 implies (4.13), since $H_{(i+1) \tau}^{+}\left(v_{1}, v_{2}\right)$ is just $H_{(i+1) \tau}^{-}\left(v_{1}, v_{2}\right)+1 / 2$, cf. the caption of Figure 6 .

To see that the column $\left(v_{1}, v_{2}\right)$ evolves independently of all the others in the interval $i \tau<t<(i+1) \tau$, note that it can be influenced only by the columns labeled $\left(w_{1}, w_{2}\right)$ with $w_{1}+w_{2}=v_{1}+v_{2}+1$. However (cf. Figure 6) in this case $H_{(i+1) \tau}^{-}\left(w_{1}, w_{2}\right)=H_{(i+1) \tau}^{-}\left(v_{1}, v_{2}\right)-2$ so that, from the induction hypothesis (cf. (4.14))

$$
\hat{s}_{t} \cap C_{w_{1}, w_{2}}(K) \subset C_{w_{1}, w_{2}}\left(H_{(i+1) \tau}^{-}\left(v_{1}, v_{2}\right)\right) .
$$

In other words, the column $\left(w_{1}, w_{2}\right)$ is too low to influence the column $\left(v_{1}, v_{2}\right)$. 
(3) $H_{(i+1) \tau}^{-}\left(v_{1}, v_{2}\right)=1$. Again one has (4.14) and one can assume by monotonicity that the columns with $w_{1}+w_{2}<v_{1}+v_{2}$ are completely full in the time interval $i \tau<t<(i+1) \tau$. The argument proceeds like in the previous case once one realizes that for $w_{1}+w_{2}=v_{1}+v_{2}+1$ one has $H_{i \tau}^{+}\left(w_{1}, w_{2}\right)=1 / 2$, so that by the induction hypothesis $\hat{s}_{t} \cap C_{w_{1}, w_{2}}(K) \subset C_{w_{1}, w_{2}}(1 / 2)$ for $t \geq i \tau$ and such column cannot influence the one labeled $\left(v_{1}, v_{2}\right)$.

(4) It remains to consider the case of the columns with $H_{(i+1) \tau}^{-}=0$. Define

$$
j:=\max _{0 \leq w_{1}<K, 0 \leq w_{2}<K}\left\{w_{1}+w_{2}: H_{(i+1) \tau}^{-}\left(w_{1}, w_{2}\right)>0\right\}+1<2 K-1,
$$

with the convention that $j:=0$ if the set is empty. It is convenient to distinguish two sub-cases:

(a) if $v_{1}+v_{2}>j+1$ then, by definition of $S_{t}^{+}$one sees that $H_{i \tau}^{+}\left(v_{1}, v_{2}\right)=0$, so that (4.13) follows (both sets are empty) from the induction hypothesis (4.12).

(b) if $j \leq v_{1}+v_{2} \leq j+1$ then by monotonicity we can assume that all columns with $w_{1}+w_{2}<j$ are completely full in the time interval $i \tau \leq t \leq(i+1) \tau$, and on the other hand we know that all columns with $w_{1}+w_{2}>j+1$ are completely empty for $t \geq i \tau$. Also, we know from (4.12) that, always for $t \geqslant i \tau,\left[\hat{s}_{t} \cap C_{w_{1}, w_{2}}(K)\right] \subset C_{w_{1}, w_{2}}(2)$ for all $\left(w_{1}, w_{2}\right)$ such that $w_{1}+w_{2} \in$ $\{j, j+1\}$. We can therefore apply Lemma 4.4 to deduce that for all times $t \geqslant(i+1) \tau$ the columns with $w_{1}+w_{2}=j+1$ are completely empty, while if $w_{1}+w_{2}=j$ then $\left[\hat{s}_{t} \cap C_{w_{1}, w_{2}}(K)\right] \subset B^{-}\left(w_{1}, w_{2}, 0\right)$. Recalling the definition of $S_{t}^{+}$(in particular, as explained in the caption of Fig. 6) we have therefore proven (4.13) for all columns $\left(w_{1}, w_{2}\right)$ such that $w_{1}+w_{2} \in\{j, j+1\}$.

4.2. The general case. Here we prove Theorem 1.2 in the general situation where $\xi$ is not the maximal configuration in $\Omega_{1, n}^{h}$. The proof is conceptually similar to the one where the ceiling is maximal, and therefore some arguments will be only sketched (see however Remark 4.6 below, where an important difference between the two cases is pointed out).

As in section 4.1, we can assume by monotonicity that $h=0, n=2 M, k=M$ and $\sigma=\vee$. First of all, it is important to realize that the maximal configuration (ground state) $\left\{\eta^{(j)}=\xi\right.$ for all $\left.j=1, \ldots, M\right\}$ corresponds to the subset of $\mathcal{C}_{M}$ defined by the property that the elementary cube labeled $r=\left(r_{1}, r_{2}, r_{3}\right)$, with $r_{i} \in\{0, \ldots, M-1\}$ (cf. Section 4.1.1) belongs to $s^{-}$if and only if

$$
\xi_{M-r_{1}+r_{2}}<M-r_{1}-r_{2}
$$

(we still call the ground state $s^{-}$, even if it is no longer the empty set as in previous section). We note also that the equilibrium measure (1.4) is given in this case by

$$
\mu^{\xi, \vee}(s)=\frac{e^{-2 \alpha\left|s \backslash s^{-}\right|}}{\sum_{s^{\prime} \supset s^{-}} e^{-2 \alpha\left|s^{\prime} \backslash s^{-}\right|}} \mathbf{1}_{s^{\prime} \supset s^{-}} .
$$

Of course, the sum in the denominator is only over the configurations $s^{\prime} \subset \mathcal{C}_{M}$ which are given by unions of elementary cubes. The dynamics $s_{t}$ just coincides with that 
described in Section 4.1.1, conditionally on the event that $s_{t} \supset s^{-}$for every $t$ (of course, the initial condition $s_{t=0}$ has to verify the same property).

As in Section 4.1.2, we cut $\mathcal{C}_{M}$ into $K^{3}$ blocks $B_{v}$ of side $(\log M)^{2} \in \mathbb{N}$. Consider the dynamics started from some $s_{t=0} \supset s^{-}$. The basic estimate which allows to prove Theorem 1.2 is the following:

Proposition 4.5. For every $\alpha>0$ there exists $C(\alpha)<\infty$ (independent of $M, \xi$ and $\left.s_{t=0}\right)$ such that the following holds w.l.p.:

$$
s_{t} \subset\left(s^{-} \cup A_{1}\right)
$$

for every $(6 / 7) C(\alpha) M(\log M)^{6} \leqslant t \leqslant M^{2}$ where $A_{1}=A_{2} \cup A_{3}$,

$$
A_{2}:=\cup\left\{B_{v}: v_{3}=0, B_{v} \cap s^{-} \text {contains at least one elementary cube }\right\}
$$

and

$$
A_{3}:=\cup\left\{B_{v}: v_{3}=0, B_{\left(v_{1}-1, v_{2}, 0\right)} \notin\left(\mathcal{C}_{M} \backslash A_{2}\right) \text { and } B_{\left(v_{1}, v_{2}-1,0\right)} \notin\left(\mathcal{C}_{M} \backslash A_{2}\right)\right\},
$$

see Figure 9 .

Note that, in the case $\xi=\wedge$, one has $A_{2}=\emptyset, A_{3}=B_{(0,0,0)}$ and Proposition 4.5 follows from Theorem 4.2 above.

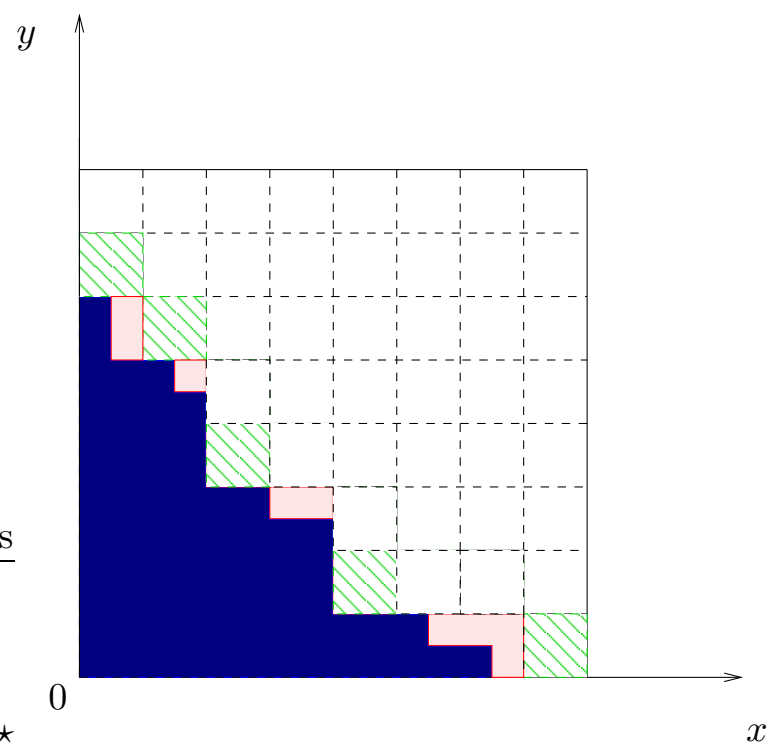

Figure 9. The sets $A_{i}$ and $s^{-}$seen from above. Squares should be imagined to have side $(\log M)^{2}$, and here $K=M /(\log M)^{2}=8$. The dark region is a horizontal section of $s^{-}$, while the lightly colored (respectively, the dashed) region is the set $A_{2} \backslash s^{-}$(resp. $A_{3}$ ) seen from above. $s^{-}$extends vertically up to height $M$, while $A_{2}, A_{3}$ extend only up to height $(\log M)^{2}$ (i.e., one single block). Note that the block $B_{(0,6,0)}$ belongs to $A_{3}$ because neither $B_{(-1,6,0)}$ nor $B_{(0,5,0)}$ belong to $\mathcal{C}_{M} \backslash A_{2}$ (in particular, $B_{(-1,6,0)}$ falls out of $\left.\mathcal{C}_{M}\right)$. A similar remark holds for $B_{(7,0,0)}$. 
Proposition 4.5 is proven below, and now we show that it does imply Theorem 1.2 . Indeed, note that the volume of $A_{1}$ satisfies

$$
\left|A_{1} \backslash s^{-}\right| \leqslant 4 M(\log M)^{4},
$$

so that

$$
\min _{s: s^{-} \subset s \subset\left(s^{-} \cup A_{1}\right)} \mu^{\xi, \vee}\left(s \mid s^{-} \subset s \subset\left(s^{-} \cup A_{1}\right)\right) \geqslant e^{-c_{8}(\alpha) M(\log M)^{4}}
$$

for some $c_{8}>0$ (we used the fact that the number of configurations $s$ satisfying $s^{-} \subset s \subset\left(s^{-} \cup A_{1}\right)$ is smaller than

$$
2^{\left|A_{1} \backslash s^{-}\right|}:
$$

this would be the exact number of configuration if there were no monotonicity constraints on $s$ ). Call, for ease of notation,

$$
T_{M}:=C(\alpha) M(\log M)^{6} .
$$

From (1.13), (4.22) and Theorem 1.1 one easily deduces that the mixing time of the dynamics constrained to $s^{-} \subset s_{t} \subset\left[s^{-} \cup A_{1}\right]$ is $O\left(M(\log M)^{4}\right)$. Since $(6 / 7) T_{M}+$ $M(\log M)^{4} \ll T_{M}$, we have proven Theorem 1.2 (with $\left.c=6\right)$.

Remark 4.6. It is important to notice that we have not proven that the dynamics started from an arbitrary $s_{t=0}$ hits the ground state $s^{-}$within time $T_{M}$, which is in general false if the ceiling $\xi$ has a jagged shape.

4.2.1. Proof of Proposition 4.5. By monotonicity, it is clear that it suffices to prove the claim for $s_{t=0}=\mathcal{C}_{M}$. As in the proof of Theorem 4.1, we introduce two deterministic subsets $\hat{S}_{t}^{ \pm}$of $\mathcal{C}_{M}$. If $S_{t}^{ \pm}$are the sets which were defined in Section 4.1.2, then we establish that

$$
\hat{S}_{t}^{-}:=S_{t}^{-} \cup s^{-},
$$

while

$$
\hat{S}_{t}^{+}:=S_{t}^{+} \cup A_{1} \text {. }
$$

We note that from the discussion of the properties of $S_{t}^{ \pm}$in Section 4.1.2 it follows that for $t \geqslant(6 / 7) T_{M}$ one has $\hat{S}_{t}^{-}=s^{-}$and $\hat{S}_{t}^{+}=A_{1}$. The claim of the proposition then follows if we can prove, in analogy with Theorem 4.2, that w.l.p. one has

$$
s_{t} \subset \hat{S}_{t}^{+}
$$

for every $0 \leqslant t \leqslant M^{2}$. The proof of this fact proceeds with the help of an auxiliary dynamics $\hat{s}_{t}$, which dominates $s_{t}$, and whose law is that of $s_{t}$ conditioned on the event that $\left\{s_{t} \supset \hat{S}_{t}^{-}\right.$for every $\left.t \geqslant 0\right\}$. Since the proof is very similar to that of Theorem 4.2, we do not give details. The only fact which requires some care is that we cannot apply Lemma 4.3 to the blocks which have a non-empty intersection with $s^{-}$, since a certain number of its elementary cubes are frozen to be full for all times.

The extra result we need concerns therefore the evolution of a single cube of side $(\log M)^{2}$ and with arbitrary ceiling. Let $\tilde{\xi} \in \Omega_{1,2(\log M)^{2}}^{0}$, let $\tilde{s}^{-} \subset \mathcal{C}_{(\log M)^{2}}=B_{(0,0,0)}$ 
be the ground state corresponding to the ceiling $\tilde{\xi}$ and $\tilde{s}_{t}$ be the evolution started from the full configuration $B_{(0,0,0)}$ and constrained to

$$
\tilde{s}^{-} \subset \tilde{s}_{t} \subset B_{(0,0,0)}
$$

for every $t>0$, and call $\tilde{T}_{m i x}$ its mixing time. Needless to say, its invariant measure is $\mu^{\tilde{\xi}, \vee}$. Notice that when $\tilde{s}^{-}=B_{(0,0,0)}$ (i.e., when $\tilde{\xi}=\vee$ ) the dynamics is trivial $\left(\tilde{s}_{t}=B_{(0,0,0)}\right.$ for all times) while when $\tilde{s}^{-}=\emptyset$ (i.e., when $\tilde{\xi}=\wedge$ ) the forthcoming lemma is already implied by Lemma 4.3 (just replace $M$ with $(\log M)^{2}$ there).

Lemma 4.7. For every $\alpha>0$ there exist $C_{4}(\alpha)<\infty$ and $C_{5}(\alpha)>0$ such that uniformly in $M$ and $\tilde{\xi}$

$$
\tilde{T}_{m i x} \leqslant C_{4}(\alpha)(\log M)^{6} .
$$

Moreover, with $\tau$ defined as in (4.4),

$$
\mathbb{P}\left(\exists \tau \leqslant t \leqslant M^{2}:\left(\tilde{s}_{t} \backslash \tilde{s}^{-}\right) \cap B_{(0,0,0)}^{+} \neq \emptyset\right) \leqslant e^{-C_{5}(\alpha)(\log M)^{2}} .
$$

What Eq. (4.28) is saying is essentially that for all times larger than $(\log M)^{2} \tilde{T}_{m i x}$ but smaller than $M^{2}$ the upper half of the cube under consideration contains only the elementary cubes which are imposed by the constraint $\tilde{s}^{-} \subset \tilde{s}_{t}$. Useless to say, this means that w.l.p. the event in the left-hand side of (4.28) does not occur.

Proof of Lemma 4.7 The proof of (4.27) is completely analogous to that of (4.7) and uses the fact that the spectral gap of the dynamics is positive, uniformly in $M$ and $\tilde{\xi}$. To prove (4.28), let us recall the well known inequality which relates the total variation distance from equilibrium of a reversible Markov Chain at time $t$ with its mixing time $T_{\text {mix }}$ :

$$
\sup _{s \in \Omega}\left\|P_{t}^{s}(\cdot)-\mu(\cdot)\right\|_{\text {var }} \leqslant e^{-\left\lfloor t / T_{\text {mix }}\right\rfloor},
$$

where $\Omega$ is the state space of the Markov Chain, $\mu$ its invariant measure and $P_{t}^{s}$ the law at time $t$, if the initial condition at time zero is $s$. Call $t_{i}, 1 \leqslant i \leqslant \zeta$ the random times when Markov Chain updates occur in the time interval $\left[\tau, M^{2}\right]$, and observe that in our case $\zeta$ is a Poisson random variable of parameter $\left(M^{2}-\tau\right)(\log M)^{4}$. One has then, using (4.10), that the left-hand side of (4.28) is upper bounded by

$$
e^{-M^{2}}+\mathbb{P}\left[\zeta \leqslant 4 M^{2}(\log M)^{4} \text { and } \exists i \leqslant \zeta:\left(\tilde{s}_{t_{i}} \backslash \tilde{s}^{-}\right) \cap B_{(0,0,0)}^{+} \neq \emptyset\right] .
$$

On the other hand, calling $\tilde{P}_{t}(\cdot)$ the law of $\tilde{s}_{t}$ and defining the set

$$
U:=\left\{s \subset B_{(0,0,0)}:\left(s \backslash \tilde{s}^{-}\right) \cap B_{(0,0,0)}^{+} \neq \emptyset\right\},
$$

one has

$$
\begin{aligned}
\mathbb{P}\left[\left(\tilde{s}_{t} \backslash s^{-}\right) \cap B_{(0,0,0)}^{+} \neq \emptyset\right] & =\tilde{P}_{t}(U) \leqslant\left|\tilde{P}_{t}(U)-\mu^{\tilde{\xi}, \vee}(U)\right|+\mu^{\tilde{\xi}, \vee}(U) \\
& \leqslant e^{-\left\lfloor t / \tilde{T}_{m i x}\right\rfloor}+e^{-C_{5}(\alpha)(\log M)^{2}} .
\end{aligned}
$$

In the last step, we used (4.29) for the first term and Lemma 2.2 to estimate the equilibrium probability of $U$. The claim (4.28) then follows from (4.30), a union bound and the fact that $\tau / \tilde{T}_{\text {mix }} \geqslant C_{6}(\alpha)(\log M)^{2}$ for some $C_{6}(\alpha)>0$. 


\section{ACKNOWLEDGMENTS}

F.L.T. was partially supported by ANR, project POLINTBIO and project LHMSHE. P.C. was partially supported by NSF Grant DMS-0301795. F.M. was partially supported by the Advanced Research Grant "PTRELSS" ADG-228032 of the European Research Council.

\section{REFERENCES}

[1] R. Bubley and M. Dyer, Path coupling: A technique for proving rapid mixing in Markov chains, Proc. of the 38th Annual Symposium on Foundations of Computer Science, pp. 223-231 (1997).

[2] R. Cerf, R. Kenyon, The low-temperature expansion of the Wulff crystal in the 3D Ising model, Comm. Math. Phys. 222 (2001), 147-179.

[3] H. Cohn, M. Larsen, J. Propp, The shape of a typical boxed plane partition, New York J. Math. 4 (1998), 137-165.

[4] P. Caputo, F. Martinelli, Asymmetric diffusion and the energy gap above the 111 ground state of the quantum XXZ model, Comm. Math. Phys. 226 (2002), 323-375.

[5] P. Caputo, F. Martinelli, F.L. Toninelli, On the approach to equilibrium for a polymer with adsorption and repulsion, Electronic Journal of Probability 13 (2008), 213-258.

[6] S. Greenberg, A. Pascoe, D. Randall, Sampling biased lattice configurations using exponential metrics, Proc. of the 19th Annual ACM-SIAM Symposium on Discrete Algorithms, pp. 76-85 (2009).

[7] R. Kenyon, Lectures on dimers, available at www.math.brown.edu/ rkenyon/papers/index.html

[8] D. A. Levin, Y. Peres, E. L. Wilmer, Markov Chains and Mixing Times, American Mathematical Society (2008).

[9] D. B. Wilson, Mixing times of Lozenge tiling and card shuffling Markov chains, Ann. Appl. Probab. 14 (2004), 274-325.

[10] E. M. Wright, Asymptotic partition formula, I. Plane partitions, Quart. J. Math. 2 (1931), 177-189.

Dipartimento di Matematica, Università Roma Tre, Largo S. Murialdo 1, 00146 Roma, Italia, and UCLA Mathematics Department Box 951555 Los Angeles, CA 90095-1555. EMAIL: caputo@mat.uniroma3.it

Dipartimento di Matematica, Università Roma Tre, Largo S. Murialdo 1, 00146 Roma, ITALIA. E-MAIL: martin@mat.uniroma3.it

CNRS and EnS Lyon, Laboratoire de Physique, 46 Allée d'Italie, 69364 Lyon, France. E-MAIL: fabio-lucio.toninelli@ens-lyon.fr 